\title{
Writers and Readers of Histone Acetylation: Structure, Mechanism, and Inhibition
}

\author{
Ronen Marmorstein ${ }^{1}$ and Ming-Ming Zhou ${ }^{2}$ \\ ${ }^{1}$ Program in Gene Expression and Regulation, Wistar Institute, and Department of Chemistry, University of \\ Pennsylvania, Philadelphia, Pennsylvania, 19104; ${ }^{2}$ Department of Structural and Chemical Biology, Icahn School \\ of Medicine at Mount Sinai, New York, New York 10065
}

Correspondence: marmor@wistar.org

\section{SUMMARY}

Histone acetylation marks are written by histone acetyltransferases (HATs) and read by bromodomains (BrDs), and less commonly by other protein modules. These proteins regulate many transcription-mediated biological processes, and their aberrant activities are correlated with several human diseases. Consequently, small molecule HAT and BrD inhibitors with therapeutic potential have been developed. Structural and biochemical studies of HATs and BrDs have revealed that HATs fall into distinct subfamilies containing a structurally related core for cofactor binding, but divergent flanking regions for substratespecific binding, catalysis, and autoregulation. BrDs adopt a conserved left-handed four-helix bundle to recognize acetyllysine; divergent loop residues contribute to substrate-specific acetyllysine recognition.

\section{Outline}

1 Introduction to writers, erasers, and readers of histones

2 Histone acetyltransferases

3 Acetyllysine readers
4 Perspectives

References

Editors: C. David Allis, Marie-Laure Caparros, Thomas Jenuwein, and Danny Reinberg

Additional Perspectives on Epigenetics available at www.cshperspectives.org

Copyright (C) 2014 Cold Spring Harbor Laboratory Press; all rights reserved; doi: 10.1101/cshperspect.a018762

Cite this article as Cold Spring Harb Perspect Biol 2014;6:a018762 


\section{OVERVIEW}

Histone acetyltransferases (HATs), sometimes referred to as lysine acetyltransferases or KATs, form a superfamily of enzymes that acetylate the side-chain amino group of lysine residues on histones, and in some cases also other proteins. These enzymes contribute to several different transcriptionmediated biological processes including cell-cycle progression, dosage compensation, and hormone signaling. Aberrant HAT function is correlated with several human diseases, including leukemic translocations, solid tumors, and metabolic disorders. In addition, protein acetylation reaches beyond histones and transcription-associated biological processes to other cellular processes, based on recent proteomic studies.

The acetylation marks on lysine residues are read by small protein modules called bromodomains (BrDs), sometimes referred to as "readers." These domains are conserved within many chromatin-associated proteins including some HATs, as well as other posttranslational modification enzymes (sometimes referred to as "writers") and ATP-dependent remodeling proteins. More recently, a PHD finger, previously shown to target methylated lysine residues, was also shown to bind acetyllysine (Kac), opening up the possibility that other types of domains may also read acetyllysine marks. Currently, many BrD-containing proteins do not have well-characterized functions, although some have been implicated in diseases such as inflammation, viral infection, solid tumors, and leukemias.

Structural and biochemical studies on HATs and BrDs have provided important mechanistic insights into the function of these writers and readers of histone acetylation. Five well-studied HAT subfamilies include Hat1 (or KAT1 according to the Allis et al. 2007 nomenclature), Gcn5/PCAF (KAT2A/KAT2B), MYST (KAT5), p300/CBP (KAT3B/KAT3A), and Rtt109 (KAT11). These HAT enzyme subfamily writers perform similar overall chemistry and have structurally related core regions that template substrates in an analogous fashion; however, they fall into subfamilies with very limited to no sequence homology. Consequently, they contain structurally divergent core flanking regions, which mediate divergent mechanisms of catalysis and possibly different modes of substrate recognition and regulation. Many HATs are regulated by autoacetylation. Inhibition of HAT enzymes by small molecule compounds is in the very early stages of development, but the prospects for exploiting HATs as therapeutic targets are strong.

The BrD readers adopt a conserved left-handed four-helix bundle and possess conserved residues within interhelical loops that recognize acetyllysine. Other residues flanking either side of the acetylated lysine contribute to binding specificity. Interestingly, many bromodomains come in multiples, and many have divergent functions such as binding two or more acetyllysine residues simultaneously or, in some cases, may have other functions distinct from acetyllysine recognition. Given the association of BrD-containing proteins with disease, there has been considerable interest in developing BrD inhibitors. Remarkably, several potent and selective inhibitors have already been developed that look promising for therapeutic applications. 


\section{INTRODUCTION TO WRITERS, ERASERS, AND READERS OF HISTONES}

DNA within the eukaryotic nucleus is compacted into chromatin containing the histone proteins $\mathrm{H} 1, \mathrm{H} 2 \mathrm{~A}$, $\mathrm{H} 2 \mathrm{~B}, \mathrm{H} 3, \mathrm{H} 4$. The appropriate regulation of chromatin orchestrates all DNA-templated reactions such as DNA transcription, replication, repair, mitosis, and apoptosis (Williamson and Pinto 2012). The macromolecules that regulate chromatin fall into distinct classes of molecules. These include ATP-dependent remodeling proteins that mobilize the histones within chromatin (Becker and Workman 2013), histone chaperones that insert and remove generic or variant histones into chromatin (covered in Almouzni and Cedar 2014), posttranslational modification enzymes that add and remove chemical groups to the DNA or histone components of chromatin (Bannister and Kouzarides 2011), chromatin recognition proteins that specifically recognize DNA, histones or modified histone, or DNA (Yap and Zhou 2010; Glatt et al. 2011), and noncoding RNA molecules that bind and modulate chromatin regulatory proteins (Mattick and Makunin 2006; Kurth and Mochizuki 2009). These macromolecules work in a highly coordinated fashion to regulate distinct chromatin templated activities.

The posttranslational modification (PTM) enzymes include proteins that add chemical groups as well as those that remove them. The enzymes that mediate histone modification (i.e., writers) include acetyltransferases, methyltransferases, kinases, and ubiquitinases. The enzymes that remove these modifications (i.e., erasers) include deacetylases, phosphatases, demethylases, and deubiquitinases (Bannister and Kouzarides 2011). Protein domains have also been identified that can recognize specific histone modifications (i.e., readers), although there appears to be more flexibility than the enzymes that create the modifications (Yap and Zhou 2010; Glatt et al. 2011). For example, bromodomains selectively target acetyllysine residues, whereas many chromodomains bind methylated lysines, and tudor domains bind methylated arginines. However, methylated lysines are also recognized by PHD fingers, WD40 domains, and ankyrin repeats (Brent and Marmorstein 2008). Many of these protein domains recognize unmodified histones as well.

Of the enzymes that perform posttranslational modification on histones, the enzymes that mediate lysine acetylation and deacetylation were the first identified. In 1996, Allis and coworkers purified a histone acetyltransferase (HAT) from Tetrahymena thermophila that was orthologous to a previously identified transcriptional adaptor from yeast called Gcn5 and conserved from yeast to man (Brownell et al. 1996). During the same time, Sternglanz and coworkers (Kleff et al. 1995) and Gottschling and coworkers (Parthun et al. 1996) also identified a histone acetyltransferase called HAT1 that was initially proposed to be a cytoplasmic specific acetyltransferase and later shown to also harbor nuclear functions (Ruiz-Garcia et al. 1998; Ai and Parthun 2004; Poveda et al. 2004). In the same year, Schreiber and colleagues isolated a mammalian histone deacetylase (HDAC) that was highly homologous to a previously characterized transcriptional repressor Rpd3 (covered in Seto and Yoshida 2014), also with conservation from yeast to man (Taunton et al. 1996). Subsequent to these groundbreaking studies, other HATs and HDACs were identified along with other types of enzymes that modify histones (Hodawadekar and Marmorstein 2007; Marmorstein and Trievel 2009). Many histone posttranslational modifications can be correlated with different DNA-templated activities. HAT and HDAC activities, however, are generally correlated with gene activation and repression/ silencing, respectively. Aberrant HAT and HDAC activities are also correlated with diseases such as cancer and metabolic disorders (Keppler and Archer 2008a; Keppler and Archer 2008b). To date, the HATs and HDACs are, biochemically and structurally, the most well-characterized of the histone PTM enzymes. This article will cover what is known to date about the structure, mechanism of action, and inhibition of HAT enzymes. Readers are directed to the collection's article on HDACs dealing with the topic of histone lysine deacetylation (Seto and Yoshida 2014, and other excellent review articles therein).

Bromodomains were the first histone modification readers that were identified when Zhou, Aggarwal, and coworkers determined the three-dimensional solution structure of the PCAF bromodomain using nuclear magnetic resonance spectroscopy. They also performed related biochemical studies revealing that it is specifically capable of binding acetyllysine modifications in peptides derived from histones H3 and H4 (Dhalluin et al. 1999). Subsequent studies by others confirmed the acetyllysine-binding properties of bromodomains from other proteins along with the identification and characterization of other types of protein domains that specifically recognize other histone modifications (Yap and Zhou 2010). Interestingly, bromodomains are found in many different types of chromatin regulators as well as protein complexes that play important roles in the human biology of health and disease. Thus, recently they have become attractive therapeutic targets, illustrated by the discovery of JQ1 and I-BET described in Qi (2014) and Schaefer (2014) (also discussed in Busslinger and Tarakhovsky 2014). In addition to describing HAT structure and function, this article will also cover what is known to date about the structure, mechanism of action, and inhibition of bromodomains. 


\section{HISTONE ACETYLTRANSFERASES}

\subsection{Classification of HATs}

Since the isolation of the Gcn5 HAT from Tetrahymena by Allis and coworkers (Brownell et al. 1996), and the identification of HAT1 by Sternglanz and coworkers (Kleff et al. 1995) and Gottschling and coworkers (Parthun et al. 1996) just more than a decade ago, many other HATs have been identified from yeast to man. Some of these HATs (e.g., PCAF and HAT1) show sequence conservation with Gcn5 within their catalytic domain, leading to their classification as Gcn5-related histone $\mathrm{N}$-acetyltransferases (GNATs; Neuwald and Landsman 1997). Many other HATs, like CBP/ p300, Rtt109, and the MYST proteins have extremely limited sequence conservation. Based on this sequence divergence within the HAT domain, HATs can be grouped into at least five different subfamilies (Table 1). This includes HAT1 (named histone acetyltransferase 1 as the founding member of the superfamily or KAT1 according to the Allis et al. 2007 nomenclature), Gcn5/PCAF (named for its founding member yeast Gcn5 and its human ortholog, PCAF, or KAT2a/KAT2B according to the alternative nomenclature), MYST (named for the founding members MOZ, Ybf2/ Sas3, Sas2, and TIP60, or KAT5), p300/CBP (named for the two human paralogs p300 and CBP, or KAT3B/KAT3A), and Rtt109 (named for its initial identi- fication as a regulator of Ty1 transposition gene product 109 , also referred to as KAT11). Although the Gcn5/PCAF, HAT1, and MYST subfamilies have homologs from yeast to man, p300/CBP is metazoan specific, and Rtt109 is fungal specific. Although other nuclear HAT subfamilies have been identified, such as the steroid receptor coactivators (ACTR/AIB1, SRC1) (Spencer et al. 1997), TAF250 (Mizzen et al. 1996), ATF-2 (Kawasaki et al. 2000), and CLOCK (Doi et al. 2006), their HAT activities have not been studied as extensively as the five major HAT classes and will not be further discussed here.

\subsection{Overall HAT Structure}

Representative structures of each of the five subfamilies of HAT proteins have been determined by X-ray crystallography, revealing the molecular characteristics of the enzymatic domains and molecular insights into catalysis and substrate acetylation.

Yeast histone acetyltransferase HAT1 (yHAT1) was the first HAT structure to be reported (Dutnall et al. 1998), setting the stage for structural analysis of this superfamily of enzymes. The structure of HAT1 bound to its AcCoA cofactor consists of an elongated $\alpha-\beta$ structure (Fig. 1A). The structure contains a conserved core region containing a three-stranded $\beta$-sheet and a long helix in parallel, span-

Table 1. The five major HAT families

\begin{tabular}{|c|c|c|}
\hline $\begin{array}{l}\text { Major HAT } \\
\text { subfamilies }\end{array}$ & $\begin{array}{l}\text { Prominent } \\
\text { members }\end{array}$ & Key structural and biochemical properties \\
\hline HAT1 & yHat1 & $\begin{array}{l}\text { Member of the GNAT family } \\
\text { Amino- and carboxy-terminal segments used for histone substrate binding } \\
\text { Requires the yHat } 2 \text { regulatory subunit for maximal catalytic activity }\end{array}$ \\
\hline Gcn5/PCAF & $\begin{array}{l}\text { yGcn5 } \\
\text { hGCN5 } \\
\text { hPCAF }\end{array}$ & $\begin{array}{l}\text { Member of the GNAT family } \\
\text { Uses a ternary complex catalytic mechanism } \\
\text { Amino- and carboxy-terminal segments used for histone substrate binding }\end{array}$ \\
\hline MYST & $\begin{array}{l}\text { yEsa1 } \\
\text { ySas2 } \\
\text { ySas3 } \\
\text { hMOZ } \\
\text { dMof } \\
\text { hMOF } \\
\text { hTIP60 } \\
\text { hHBO1 }\end{array}$ & $\begin{array}{l}\text { Uses a ping-pong catalytic mechanism } \\
\text { Requires autoacetylation of a specific lysine at the active site for cognate histone acetylation }\end{array}$ \\
\hline p300/CBP & $\begin{array}{l}\text { hp300 } \\
\text { hCBP }\end{array}$ & $\begin{array}{l}\text { Metazoan-specific, but shows structural homology with yRtt109 } \\
\text { Uses a ternary Theorell-Chance (hit-and-run) catalytic mechanism } \\
\text { Contains a substrate-binding loop that participates in AcCoA and lysine binding } \\
\text { Contains an autoacetylation loop that requires lysine autoacetylation for maximal catalytic activity }\end{array}$ \\
\hline Rtt109 & yR11109 & $\begin{array}{l}\text { Fungal-specific, but shows structural homology with p300 } \\
\text { Contains a substrate-binding loop that participates in AcCoA and probably also lysine binding } \\
\text { Requires autoacetylation of a lysine residue near the active site for maximal catalytic activity } \\
\text { Requires one of two histone chaperone cofactors (Asfl or Vps75) for maximal catalytic activity and } \\
\text { histone substrate specificity }\end{array}$ \\
\hline
\end{tabular}

y, yeast; h, human; GNAT, Gcn5-related $N$-acetyltransferase. 
A

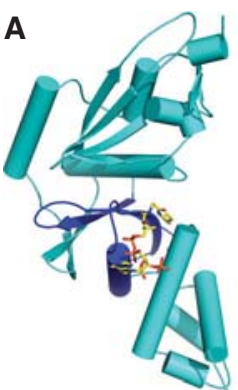

$\mathrm{yHat} 1+\mathrm{AcCoA}$

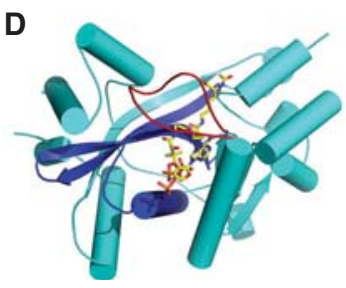

hp300 + LysCoA

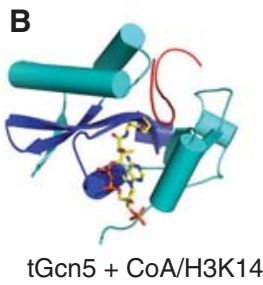

C

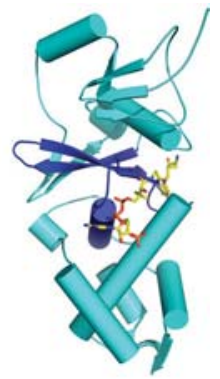

yEsa1 + H4K16CoA

E

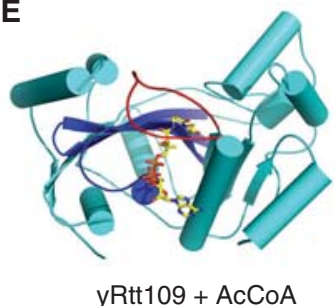

Figure 1. Overall structure of HAT proteins. Representative members of the five HAT subfamilies are illustrated as cartoons highlighting the structurally conserved core region (blue) and flanking aminoand carboxy-terminal regions (aqua). The cofactor is shown in stick figure in CPK coloring (carbon, yellow; oxygen, red; nitrogen, blue; phosphorous, orange; sulfur, brown): (A) yeast HAT1/AcCoA (PDB 1BOB), (B) Tetrahymena Gcn5/CoA/histone H3 (PDB code: 1PUA) with the histone $\mathrm{H} 3$ peptide shown in red, $(C)$ yeast Esa1/H4K16CoA (PDB code: 3TO6), (D) human p300/Lys-CoA (PDB code: $3 \mathrm{BIY}$ ) with the substrate-binding loop shown in red, $(E)$ yeast Rtt109/CoA (PDB code: 3D35) with the substrate-binding loop shown in red.

ning one side of the sheet (Neuwald and Landsman 1997). The AcCoA cofactor packs and makes interactions along one edge of this core region. The core region is flanked by a $\beta$ - $\alpha$-loop segment on one side (see top part of Fig. 1A) and an $\alpha$-rich segment on the other (see bottom part of Fig. 1A) that together form a cleft over the central core domain for the histone substrate to bind and catalysis to occur.

To date, several Gcn5 crystal structures in various liganded forms (Rojas et al. 1999; Trievel et al. 1999; Poux et al. 2002; Clements et al. 2003; Poux and Marmorstein 2003), a Gcn5/CoA solution structure (Lin et al. 1999), and the human PCAF/AcCoA structure (Clements et al. 1999) have been reported. Each of the HAT domains shows a high degree of superposition. The Tetrahymena ternary structure of a Gcn5/CoA/histone $\mathrm{H} 3$ complex reveals a core region with structural conservation with yHat 1 that make analogous AcCoA cofactor interactions, but structurally divergent amino- and carboxy-terminal regions flank the core region (Fig. 1B). The cleft over the core domain is deeper than yHat 1 and accommodates the bound histone $\mathrm{H} 3$ peptide (red loop in Fig. 1B) that is contacted largely by residues from the amino- and carboxy-terminal segments.
Several structures have also been reported of HATs from the MYST subfamily from yeast to man, including Drosophila MOF bound to AcCoA (Akhtar and Becker 2001), hMOF in unliganded form (Yuan et al. 2012), hMOZ bound to AcCoA (Holbert et al. 2007), and yEsal in various liganded forms (Yan et al. 2000; Yan et al. 2002; Yuan et al. 2012). Each of the structures superimpose well and show a structurally conserved core region with HAT1 and Gcn5/ PCAF, but divergent amino- and carboxy-terminal segments. The structure of yeast Esal bound to a linked peptide-CoA conjugate in which residues 11-22 of histone $\mathrm{H} 4$ is linked to CoA though lysine 16 (H4K16-CoA) reveals $\alpha / \beta$ amino- and carboxy-terminal domains, with the amino-terminal domain containing a TFIIIA zinc finger fold and the carboxy-terminal segment containing a helix-turnhelix domain typically found in DNA-binding proteins and proposed to participate in nucleosomal localization (Fig. 1C) (Holbert et al. 2007). The bound H4K16-CoA bisubstrate inhibitor provides important details about how both cosubstrates bind to yEsal. The CoA portion is packed against the core domain similar to the Gcn5 and HAT complexes and the lysine portion of the inhibitor is located in the central cleft formed by the flanking amino- and carboxy-terminal segments. The rest of the $\mathrm{H} 4$ peptide is disordered in the crystal structure.

The structures of human p300 (hp300) bound to a LysCoA bisubstrate inhibitor (Liu et al. 2008), yeast Rtt109 (yRtt109) bound to CoA or AcCoA cofactors (Lin and Yuan 2008; Stavropoulos et al. 2008; Tang et al. 2008), or bound to cofactor and the Vps75 histone chaperone (Kolonko et al. 2010; Su et al. 2011; Tang et al. 2011) have been reported. Remarkably, the hp300 and yRtt109 structures show a high degree of superposition despite the absence of significant sequence homology (Fig. 1D,E). These structures reveal an elongated globular domain containing a central seven-strand $\beta$-sheet surrounded by nine $\alpha$-helices and several loops. Roughly in the center of this domain is located the structurally conserved core region that is involved in cofactor interactions, whereas regions that flank this core diverge from the other HATs. A unique feature of these HATs relative to the others is a long, approximately 25 residue loop (colored in red in Fig. 1D,E), called the substrate-binding loop, that encapsulates the cofactor in yRtt109 and the Lys-CoA bisubstrate inhibitor in hp300.

Taken together, each of the 5 HAT subfamilies shares a conserved central core region that contributes to AcCoA cofactor binding, but divergent amino- and carboxy-terminal segments that flank this core also appear to contribute to histone substrate binding. The p300 and Rtt109 HAT subfamilies (Fig. 1D,E) are structurally conserved throughout the HAT domain and contain a unique, approximately 25 residue substrate-binding loop that appears 
to participate in the binding of both AcCoA and histone substrates.

\subsection{Catalytic Mechanism}

HATs transfer the acetyl group from the acetyl-CoA cofactor to the $\mathrm{N} \zeta$ nitrogen of a lysine side chain within histones. Structural, biochemical, mutational, and enzymatic analyses have provided insights into the catalytic mechanism of these enzymes. A remarkable outcome of these studies is that each HAT subfamily uses a different catalytic strategy for acetyl transfer. This is unusual for a superfamily of enzymes that catalyze the same chemical reaction, but perhaps not so surprising for these enzymes because the transfer of an acetyl group from a thioester to an amine is not a chemically demanding reaction, thus allowing different HAT subfamilies to use different chemical strategies to mediate acetyl transfer.

Members of the Gcn5/PCAF subfamily of HATs were the first proteins of the superfamily whose enzymatic properties were characterized in detail in the context of the available crystal structures. Specifically, the crystal structures of Gcn 5 and PCAF revealed the presence of a strictly conserved glutamate in the active site (Glu173 in yGcn5 and Glu570 in hPCAF) that is in position to act as a general base for catalysis through a well-ordered water molecule in the crystal structure (Fig. 2A). The glutamate is buried in a hydrophobic pocket, which likely raises its acid dissociation constant $(\mathrm{pKa})$ for proton extraction. The importance of this glutamate residue was confirmed by mutational analysis (Tanner et al. 1999; Trievel et al. 1999) in which an E173Q mutant in yGcn5 showed a 360-fold decrease in enzymatic turnover ( $k_{\text {cat }}$; Tanner et al. 1999). Denu and coworkers also showed that Gcn5 functions through a ternary complex mechanism in which both substrates (i.e., lysine and $\mathrm{AcCoA}$ ) must be bound to the enzyme before catalysis can occur. This involves deprotonation of the lysine substrate by Glu173, facilitating the direct transfer of the acetyl group from AcCoA to the lysine side chain (Tanner et al. 1999). Denu and coworkers showed similar results for PCAF (Tanner et al. 2000), however, an acidic residue, if any, that protonates the CoA leaving group has not been identified.

The crystal structure of the yEsa1 member of the MYST subfamily of HATs revealed the presence of a glutamate residue (Glu338) that is strictly conserved within the MYST HATs. It overlays with the catalytic glutamate residue of Gcn5/PCAF, and a yEsa1 E338Q mutant only catalyzed background levels of acetylation (Yan et al. 2000). Interestingly, a subsequent structural analysis and enzymatic characterization revealed that an active site cysteine residue (Cys304), conserved within the MYST HAT sub- family, also plays an important catalytic role with C304S and C304A mutants shown to be highly defective (Fig. 2B) (Yan et al. 2002). Kinetic analysis with both substrates is consistent with a ping-pong catalytic mechanism, in which the enzyme first forms an acetylated intermediate involving Cys304 before the formation of an acetylated histone product. In this mechanism, Glu338 is proposed to serve as a general base, which deprotonates both Cys304 and the histone lysine side chain in preparation for both acetyltransfer events (Yan et al. 2002). Paradoxically, a more recent report shows that Esa1 assembled within a physiologically relevant piccolo NuA4 complex does not show a strong dependence on Cys304 for catalysis, leading the investigators to conclude that yEsal within the piccolo NuA4 complex proceeds through a ternary catalytic mechanism similar to Gcn5/PCAF (Berndsen et al. 2007). This would suggest that the same HAT enzyme may use different catalytic mechanisms within different cellular contexts.

The p300 crystal structure reveals that there is no glutamate residue that is analogous to the key residues within the Gcn5/PCAF and MYST HAT subfamilies that function as general base residues for catalysis (Liu et al. 2008). Mutagenesis and kinetic analysis of the potential catalytic residues in the active site uncovered only two residues (Tyr1467 and Trp1436) that showed a significant effect on catalysis when mutated (Fig. 2C) (Liu et al. 2008); a Y1467F substitution showed about a 400-fold reduction in catalytic efficiency $\left(k_{\mathrm{cat}} / K_{\mathrm{M}}\right)$, a W1436A substitution reduced the catalytic efficiency 50 -fold, and a W1436F substitution showed a more modest effect on catalysis. Based on the position of these residues in the structure, Tyr1467 was proposed to play a role as a general acid for catalysis, whereas Trp1436 was proposed to help orient the target lysine into the active site. These residues are strictly conserved within the p300/CBP HAT subfamily, correlating with the notion that Tyr1467 and Trp1436 are important for catalysis. Thus, it appears that p300/CBP does not use a general base for catalysis, in contrast to the Gcn5/PCAF and MYST HAT subfamilies. Taken together with the fact that hp300 is inhibited potently by a more primitive LysCoA inhibitor, but poorly by bisubstrate inhibitors with longer peptide moieties (Lau et al. 2000) and the observation that longer peptides are better substrates for p300 than lysine, it was proposed that the p300/CBP subfamily of HATs use a "hit-and-run" or Theorell-Chance acetyltransfer mechanism that is distinct from the catalytic mechanisms used by the Gen5/PCAF and MYST HAT subfamilies.

The kinetic mechanism for acetylation by Rtt109 and Hat 1 has been less well-characterized than the other HAT subfamilies. We know both enzymes require the association of other protein regulatory subunits for full activity. In the 
A

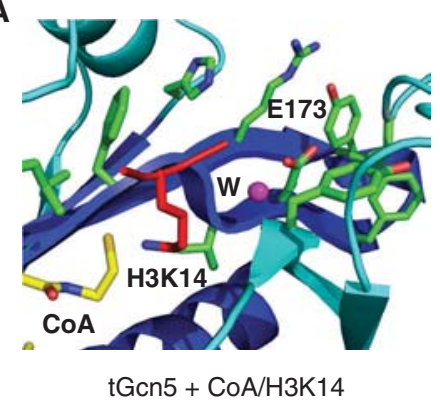

C

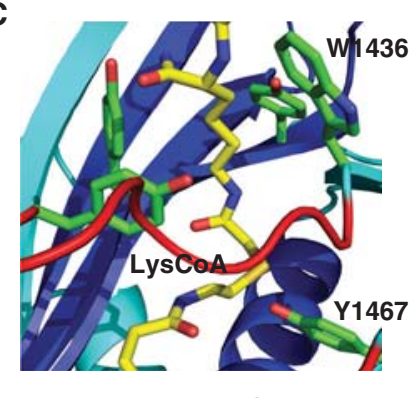

hp300 + LysCoA

E

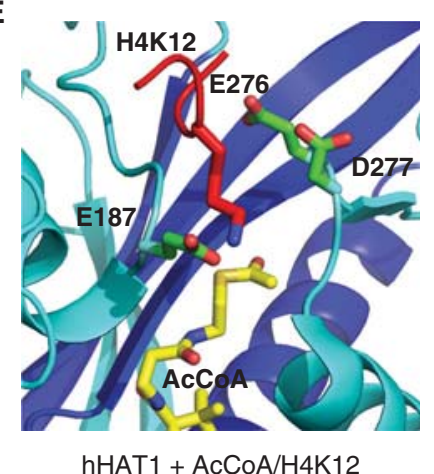

B

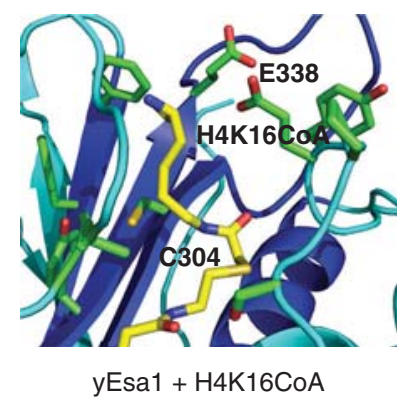

D

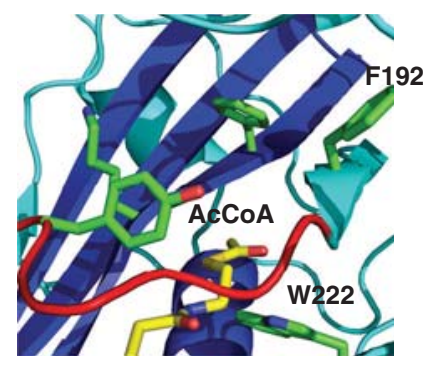

$y \mathrm{Rtt} 109+\mathrm{AcCoA}$

Figure 2. Catalytic mechanism of HAT proteins. Active sites of representative members of the HAT subfamilies are illustrated highlighting the relevant side chains on a backbone cartoon of the active site. (A) Tetrahymena Gcn5/ $\mathrm{CoA} /$ histone $\mathrm{H} 3$. Key catalytic residues are labeled and hydrophobic residues of the active site that likely raise the pKa of Glu 173 are shown in stick figure in CPK coloring with carbon in green. A segment of the histone H3 peptide is shown in red. W indicates a well-ordered water molecule that participates in catalysis. The numbering is for yeast Gcn5. (B) Yeast Esal bound to the H4K16CoA bisubstrate inhibitor (stick figure and CPK coloring with carbon atoms in yellow). Key catalytic residues are labeled and hydrophobic residues of the active site that likely raise the pKa of Glu 338 are shown. Residues flanking K16 in the peptide are disordered in the structure. $(C)$ Human p300 bound to the Lys-CoA bisubstrate inhibitor (stick figure and CPK coloring with carbon atoms in yellow). Residues shown to play catalytic roles are labeled with other potential catalytic residues shown in stick figure. The substrate-binding loop is shown in red. (D) Yeast Rtt109/CoA. Potential catalytic residues in the corresponding position of hp300 are shown. The CoA molecule is shown in stick figure in CPK coloring with carbon atoms in yellow. The substratebinding loop is shown in red. $(E)$ hHAT1/AcCoA/histone H4. The three general base candidate residues are represented as green stick figures and a segment of the histone $\mathrm{H} 4$ peptide is shown in red.

case of Rtt109, it harbors very low acetyltransferase activity on its own (Driscoll et al. 2007; Tsubota et al. 2007), but its activity is stimulated by association with either the Asf1 or Vps75 histone chaperone proteins (Han et al. 2007a; Han et al. 2007b; Tsubota et al. 2007; Berndsen et al. 2008;
Albaugh et al. 2010). Although it is not clear how these histone chaperones enhance the catalytic activity of Rtt109, the crystal structures of Rtt109/Vps75 complexes (in both 2:2 and 2:1 stoichiometries) show no significant changes in the Rtt109 active site as a function of Vps75 binding, sug- 
gesting that the histone chaperones merely function to deliver histone substrates to Rtt109 for acetylation (Kolonko et al. 2010; Su et al. 2011; Tang et al. 2011). The crystal structure of Rtt109 alone revealed that despite its overall structural superposition with the hp300 HAT domain, the key catalytic residues of hp300 (Tyr1467 and Trp1436) are not conserved in Rtt109 (Fig. 2D). Instead, kinetic analysis showed that the Rtt109/Vps75 complex uses a sequential kinetic mechanism whereby the Rtt109-Vps75 complex, AcCoA, and histone $\mathrm{H} 3$ substrates form a complex before chemical catalysis (Albaugh et al. 2010). Structural, mutational, and kinetic analysis of Rtt109 points to the importance of Asp89 and Trp222 for catalysis. The D89N and W222F mutants showed about a 25 -fold defect in catalytic rate; however, this was mostly because of a $K_{\mathrm{M}}$ effect for AcCoA binding (Tang et al. 2011), as was the 1000-fold reduced catalytic efficiency of a D288N mutant (Albaugh et al. 2010; Kolonko et al. 2010). Key residues involved in catalytic turnover, such as a general base or acid, have not been identified for Rtt109 and thus may not be present in this case.

Although a detailed enzymatic study of yHat1 has not been reported, a recent study provides important new insights (Wu et al. 2012). Specifically, the structure determination of human HAT1 (hHAT1) bound to AcCoA and a histone $\mathrm{H} 4$ peptide centered around $\mathrm{K} 12$ reveals that three residues, Glu187, Glu276, and Asp277, are in proximity to the $\mathrm{N} \zeta$ nitrogen of $\mathrm{H} 4 \mathrm{~K} 12$ and thus could act as potential general base residues for catalysis (Fig. 2E) (Wu et al. 2012). A superposition of the hHAT1 active site with yGen5 and yEsa1 (MYST subfamily) reveals that residues in these enzymes that function as a general base of catalysis (i.e., glutamates 173 and 338, respectively) superimpose with Glu276 of hHAT1 (Glu255 in yHat1; Yan et al. 2000; Wu et al. 2012), which is strictly conserved among Hat 1 orthologs. In hHAT1, mutating Glu276 to Gln causes a 28.5-fold decrease in $k_{\text {cat }}$. E187Q and D277N mutations, however, cause a more modest $\sim 15.3$ - and $\sim 8.1$-fold decrease in $k_{\text {cat }}$, respectively. E276Q and E187Q increase the pKa of ionization from 8.15 to 8.74 and 9.15, respectively, whereas D277N only increases the pKa slightly to 8.35 (Wu et al. 2012). Hat 1, however, unlike MYST HATs, does not contain a cysteine residue in the active site of the enzyme. Taken together, these observations suggest that Glu276 of hHAT1, and probably also Glu187, play a role as general base(s) for catalysis, through a ternary complex mechanism similar to the Gcn5/PCAF HAT subfamily.

A comparison of the catalytic mechanisms of the different HAT subfamilies reveals remarkable diversity in the way each subfamily mediates acetyl transfer. This is likely to be a reflection of the relatively low chemical "cost" that is required to transfer an acetyl group from a thioester to an amine, as opposed to some other more demanding chem- ical reactions such as phosphorylation, and the relatively long evolutionary time that HATs had to evolve.

\subsection{Histone Substrate Binding}

To date, the only direct molecular insights into histone binding by HATs has come from structural studies of two HAT proteins; one study provided structural information on the hHAT1 HAT domain bound to AcCoA and a histone H4 peptide centered around Lys 12 (Wu et al. 2012) whereas other studies have focused on the Tetrehymena Gen5 (tGcn5) HAT domain bound to CoA and several cognate substrate peptides including histone $\mathrm{H} 3$ (centered around Lys14; Rojas et al. 1999; Clements et al. 2003; Poux and Marmorstein 2003), histone H4 (centered around Lys8), and p53 (centered around Lys320; Poux and Marmorstein 2003). These structures reveal that the histone peptide substrates bind across a groove formed by the central core region found at the base and the amino- and carboxyterminal regions on flanking sides that mediate the majority of the interactions with the substrate peptide (Fig. $3 \mathrm{~A}, \mathrm{~B})$. A comparison of the structures of $\mathrm{tGcn} 5$ bound to different peptides revealed that 15 of the 19 residues of $\mathrm{H} 3$ are ordered in the structure rather than less than 10 residues for the $\mathrm{H} 4$ and p53 peptides, which is consistent with the $>1000$-fold greater catalytic efficiency of Gcn 5 for $\mathrm{H} 3$ over H4 and p53 (Trievel et al. 2000). These structures also revealed a more ordered and extensive protein-peptide interaction in residues carboxy-terminal to the reactive lysine, arguing that substrate interactions carboxy-terminal to the target are more important for substrate binding by the Gcn5/PCAF HAT subfamily (Fig. 3A). These interactions involve mostly hydrogen bonds to backbone residues and van der Waals interactions with side chains. Not surprisingly, the residues of $\mathrm{tGcn} 5$ that make contact to the peptide substrate are highly conserved within the Gcn5/ PCAF subfamily.

When bound to hHAT1, the H4 peptide adopts a welldefined conformation that harbors a $\beta$-turn at its amino terminus, which would otherwise be in an extended form in free H4 (Fig. 3B) (Wu et al. 2012). Two hHAT1 conserved hydrophobic residues (Trp199 and Tyr225) interact with Gly9 and Lys8 at the $\beta$-turn of the $\mathrm{H} 4$ peptide, respectively, to enforce the orientation of the substrate at the entrance of a groove ( Wu et al. 2012). The carboxyl terminus of the H4 peptide contains two positively charged residues (Arg17 and Arg19) that make extensive hydrogen-bond and charge-charge interactions with invariant residues of hHAT1 (Glu64 and Asp62). The substrate-binding groove of hHAT1 narrows where H4 Lys12 binds allowing only Gly11 and Lys12 to be accommodated. The strictly conserved Glu276 of hHAT1 also makes hydrogen-bond 


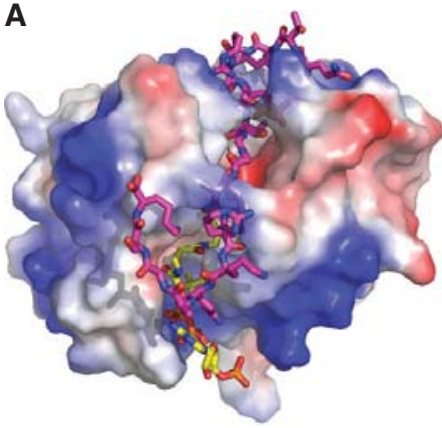

tGen5 + CoA/H3K14
B

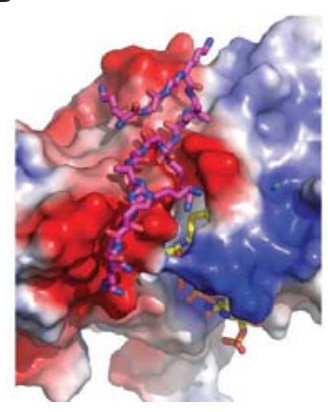

$\mathrm{hHAT} 1+\mathrm{AcCoA} / \mathrm{H} 4 \mathrm{~K} 12$
C

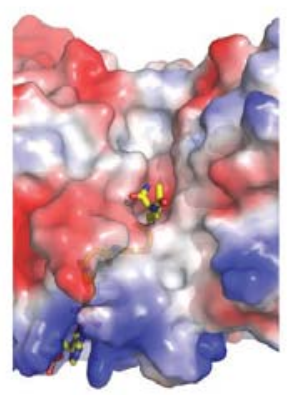

hp300 + Lys/CoA
D

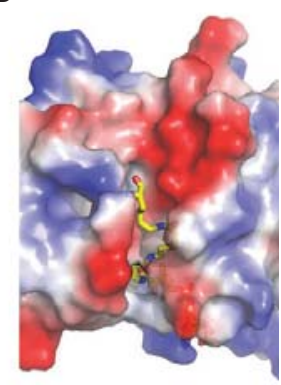

yEsa1 + H4K16/CoA

Figure 3. Histone substrate binding by HAT proteins. Close-up electrostatic view of HAT domain structures with histone peptide substrates or CoA-peptide bisubstrate inhibitors. Protein surfaces are colored according to electrostatic potential with the degree of red, blue, and white coloring correlating with electronegative, electropositive, and neutral charge, respectively. (A) Structure of tGen5 bound to CoA (CPK coloring with carbon atoms shown in yellow) and a 19-residue histone $\mathrm{H} 3$ peptide (CPK coloring with carbon atoms shown in purple) centered around K14. (B) Structure of hHAT1 bound to AcCoA (CPK coloring with carbon atoms shown in yellow) and a 20-residue histone $\mathrm{H} 4$ peptide (CPK coloring with carbon atoms shown in purple) centered around K12. (C) Structure of the hp300/LysCoA complex. The LysCoA bisubstrate inhibitor is shown in CPK coloring with carbon atoms in yellow. (D) Structure of the yEsa1/H4K16CoA complex (only the lysine side chain of the H4K16 peptide component of the bisubstrate inhibitor is ordered in the crystal structure and shown in CPK coloring with carbon atoms in yellow).

interactions with H4 Gly11 and Lys12. Together, these specific interactions explain the preference for Lys 12 of $\mathrm{H} 4$ as the acetylation target of hHAT1 (Wu et al. 2012).

The structures of human p300 (Liu et al. 2008) and the MYST HAT domain protein, yEsal (Yuan et al. 2012), crystallized with bisubstrate inhibitors has provided some information about histone substrate binding. hp300 was cocrystallized with a Lys-CoA bisubstrate inhibitor that shows an $\mathrm{IC}_{50}$ of $\sim 400 \mathrm{nM}$, and is a more potent inhibitor than peptide-CoA inhibitors, consistent with its TheorellChance catalytic mechanism. The structure of the hp300/ Lys-CoA complex revealed that the lysine portion of the bisubstrate inhibitor sits in a hydrophobic tunnel with the backbone of the lysine residue proximal to an electronegative groove on one side (Fig. 3C). This electronegative groove shown for hp300 also contains two pockets separated by a distance corresponding to about 3-4 amino acid residues. Correlating with this observation, an alignment of all known p300/CBP substrates reveals that they all contain a basic amino acid either three or four residues upstream of or downstream from the target lysine. Mutagenesis of residues that form these pockets increases the $K_{\mathrm{M}}$ for histone H3 substrate, highlighting the importance of this site for protein substrate binding by p300 (Liu et al. 2008). Taking this data together, the $\mathrm{p} 300 / \mathrm{CBP}$ HAT subfamily have more promiscuous substrate-binding properties relative to the Hat1, Gcn5/PCAF, and MYST HATs.

The yEsal HAT domain was reported crystallized with an H4K16CoA bisubstrate inhibitor (Yuan et al. 2012). Although the yEsal HAT domain and the CoA, linker, and lysine portion of the bisubstrate inhibitor is wellresolved, the rest of the peptide portion of the bisubstrate inhibitor is not and is presumed to be disordered (Fig. 3C). Like the hp300/Lys-CoA structure, the yEsa1/H4K16CoA complex shows a groove for peptide binding proximal to the lysine portion of the bisubstrate inhibitor for histone peptide substrate to bind, although this groove is more apolar than that of the hp300 HAT domain. This likely reflects the greater degree of substrate selectivity of the MYST proteins over the p300/CBP HAT subfamily.

Taken together, the structures that are available of HAT domains bound to peptide substrates or CoA-peptide bisubstrate inhibitors provide important insights into the general mode of peptide binding although the molecular details that underlie substrate-specific binding is still unclear.

\subsection{Regulation by Autoacetylation and Protein Cofactors}

Acetyltransferase activity is regulated in at least two ways, through interaction with regulatory protein subunits and through autoacetylation. Many acetyltransferases function in the context of multiprotein complexes that modulate their catalytic activity and/or substrate specificity (Carrozza et al. 2003; Lee and Workman 2007). For example, the recombinant Gcn5 and PCAF proteins are active on free histones (acetylating H3K14 and, to a lesser extent, H4K8/ K16) or histone peptides, but are much less active on nucleosomes. However, Gcn5/PCAF function in cells exclu- 
sively as multiprotein complexes and their assembly into these complexes facilitates nucleosomal acetylation and modulates intrinsic acetylation activity and substrate acetylation specificity (Carrozza et al. 2003; Lee and Workman 2007). Gcn5/PCAF-containing complexes such as SAGA/ SLIK in yeast and TFTC/STAGA in human (Nagy and Tora 2007) acetylate lysines in histone $\mathrm{H} 3, \mathrm{H} 4$, and $\mathrm{H} 2 \mathrm{~B}$ within chromatin. MYST HATs are also assembled in multiprotein complexes in cells to modulate histone acetylation in chromatin (Sapountzi and Cote 2011). For example, Esa1 is part of NuA4 and piccolo/NuA4 complexes for chromatin acetylation. It is not clear yet what the molecular basis for how associated proteins within the HAT complexes modulate the HAT activity and specificity to their respective catalytic subunits.

Several HATs require the binding of cofactors for catalytic activity. For example, the Sas2 member of the MYST HATs requires the binding of Sas 4 and Sas 5 for acetyltransferase activity (Sutton et al. 2003; Shia et al. 2005). Hat2 and Hif1 elevate yHat1 acetyltransferase activity by about 10-fold when assembled into the NuB4 complex (Parthun et al. 1996). On its own, Rtt109 shows very low activity. The histone chaperones, Vps75 or Asf1, modulate Rtt109 acetylase activity and substrate specificity by several hundredfold (Kolonko et al. 2010); the Rtt109/Vps75 complex selectively acetylates H3K9 and H3K27, whereas the Rtt109/ Asf1 complex preferentially acetylates $\mathrm{H} 3 \mathrm{~K} 56$ near the H3 core region (Driscoll et al. 2007; Han et al. 2007a; Han et al. 2007b; Tsubota et al. 2007; Tang et al. 2011). Associated subunits of Hat1 (Ruiz-Garcia et al. 1998) and Rtt109 (Tang et al. 2008; Kolonko et al. 2010; Tang et al. 2011) may contribute to catalysis, at least in part, by facilitating productive binding to the respective cognate histone substrates, based on kinetic data, but more work is required.

Another mode of regulating HAT activity that has recently come to light is the autoacetylation of HAT proteins. In particular, three HAT subfamilies have now been shown to be regulated by autoacetylation: Rtt109, p300/CBP, and MYST.

p300 contains a highly basic loop of about 40 residues embedded in the middle of its HAT domain that was shown to undergo multiple autoacetylation through a intermolecular mechanism (Karanam et al. 2007) to regulate the catalytic activity of the protein, with the hyperacetylated forms correlated with the active and hypoacetylated forms with the inactive forms of the enzyme (Thompson et al. 2004). In addition, recombinant p300 protein with a cleaved "autoacetylation loop" has been shown to be constitutively active (Thompson et al. 2004). The molecular basis for how autoacetylation regulates p300 activity is not clear because the reported p300/Lys-CoA crystal structure does not contain an intact autoacetylation loop. However, Cole and colleagues proposed a model of regulation whereby the highly basic autoacetylation loop sits in the electronegative substrate-binding site to directly compete with substrate binding. The loop would then be released from the substrate-binding site on autoacetylation for cognate substrate binding (Fig. 4A) (Liu et al. 2008).

R1109 was also known to be autoacetylated but the molecular basis for this was not known until structures of Rtt109 were reported revealing a buried acetyllysine residue (Lys290; Fig. 4B) (Lin and Yuan 2008; Stavropoulos et al. 2008; Tang et al. 2008). The acetylation of Lys290 is required for full acetylation activity (Albaugh et al. 2011). This acetyllysine at 290 forms hydrogen bonds with Asp288, a mutationally sensitive Rtt109 residue. Mass spectrometry analysis showed that this acetylation occurs in vitro as well as in yeast cells (Tang et al. 2008). Reports addressing the functional importance of Lys 290 modification have yielded contradictory results with regard to genotoxic agent sensitivity in vivo (Lin and Yuan 2008; Stavropoulos et al. 2008; Tang et al. 2008). A recent study by Denu and coworkers showed that Lys290 autoacetylation increases the overall $K_{\text {cat }}$ and decreases the $K_{\mathrm{M}}$ for AcCoA binding (Albaugh et al. 2011), although the molecular basis for this is unknown.

Several recent reports have shown that the hMOF MYST protein is autoacetylated at an active site lysine (Lys274) and that this autoacetylation is required for cognate acetyltransferase activity both in vitro and in vivo ( $\mathrm{Lu}$ et al. 2011; Sun et al. 2011; Yuan et al. 2012). The lysine that was found to be autoacetylated in hMOF is strictly conserved in MYST proteins, and Yuan et al. also showed that acetylation of this lysine residue occurs in yeast Esal and Sas2 and is required for acetylation of these proteins in vitro and for function of these enzymes in cells (Yuan et al. 2012). The structure of the yEsa1/H4K16CoA complex with this active site lysine in the acetylated form (Lys262Ac) reveals that it sits in the active site pocket with the acetyl CO group making hydrogen bonds to Tyr289 and Ser291, and the methyl group making van der Waals interactions with phenylalanines 271 and 273; the aliphatic region of Lys262Ac makes van der Waals interactions with the lysine of the H4K16CoA bisubstrate inhibitors (Fig. 4C) (Yuan et al. 2012). Each of the residues that contact the acetylated lysine is strictly or highly conserved among the entire MYST protein subfamily further arguing for the importance of this acetylated lysine in MYST function. The structure of the hMOF HAT domain has also been determined in unliganded form (Yuan et al. 2012). This structure reveals that Lys274 exists in two states. In one state it is acetylated and in the same conformation as Lys262Ac of yEsal, making analogous intra-atomic interactions. In a second state, Lys 274 is unacetylated and flipped by about $90^{\circ}$ out of the 


\section{A}

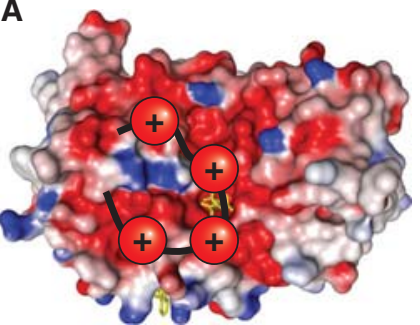

p300

B

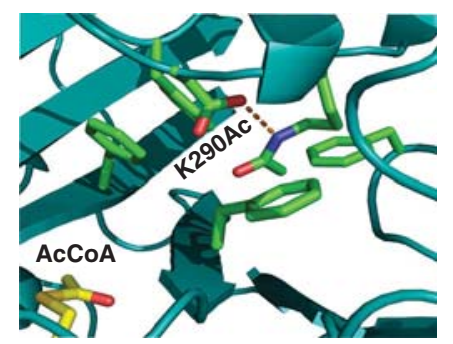

Rtt109

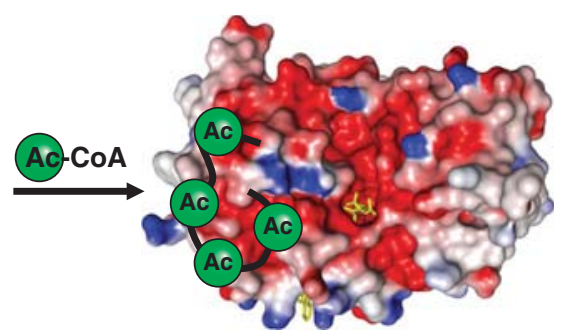

C

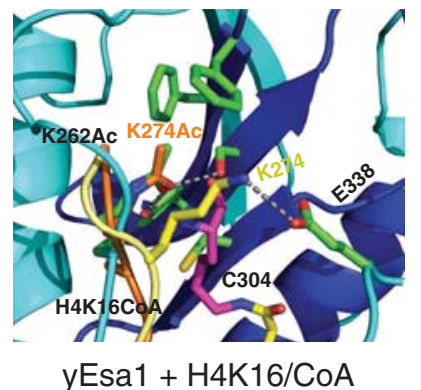

Figure 4. Autoacetylation regulation of HAT proteins. Close-up views of the autoacetylation site of HAT proteins. (A) Model for p300 activation by autoacetylation. The black loop and green acetylated lysine balls are modeled on the p300/Lys-CoA crystal structure. (B) Structure of the K290 autoacetylation site of Rtt109, highlighting the environment around acetylated K290. The acetylated lysine and other side chains that interact with the acetylated lysine are indicated in stick figure in CPK coloring with carbon in green and the hydrogen bond is shown as a dotted orange line. The AcCoA molecule is shown as a stick figure in CPK coloring with carbon atoms in yellow. $(C)$ Structure of the yEsa1/H4K16CoA complex is shown, highlighting the environment around acetylated K262 (green). The corresponding K274 of hMOF is superimposed in the unacetylated (yellow) and acetylated (orange) conformations showing that the unacetylated conformation would clash with binding of the cognate substrate lysine (as represented by the lysine of the H4K16CoA bisubstrate inhibitor shown in purple).

active site, such that the lysine forms a long hydrogen bond with Glu350, the general base for catalysis. This position would block cognate lysine binding (Fig. 4C). This is consistent with biochemical studies that show that a $\mathrm{K}$ to $\mathrm{R}$ mutant of this lysine in hMOF is defective in cognate substrate binding (Yuan et al. 2012). Taken together, these structural, biochemical, and functional studies show that autoacetylation of MYST proteins in the active site is required for cognate substrate acetylation.

\subsection{Inhibitors of Acetyltransferases and Implications for Drug Development}

HATs mediate many different biological processes including cell-cycle progression, dosage compensation, repair of DNA damage, and hormone signaling. Aberrant HAT function is correlated with several human diseases including solid tumors, leukemias, inflammatory lung disease, viral infection, diabetes, fungal infection, and drug addiction (Heery and Fischer 2007; Renthal and Nestler 2009). The p300/CBP HAT has properties of both an oncoprotein and tumor suppressor protein. As an oncoprotein, the CBP
HAT forms translocation products with MLL (mixed lineage leukemia) and MOZ (monocytic leukemia zinc-finger protein), another HAT, in a subset of acute myeloid leukemias. When mutated, the p300 HAT is found in a subset of colorectal and gastric cancers making it a bonafide tumor suppressor. The activities of HATs and HDACs are also changed in asthma and chronic obstructive pulmonary disease because bronchial biopsies and alveolar macrophages from asthmatic patients show increased HAT and reduced HDAC activity (Barnes et al. 2005). p300-mediated acetylation of the HIV-1 viral protein, integrase, increases its activity in integrating the HIV-1 virus into the human genome (Cereseto et al. 2005). The leading diabetes drug, Metformin, was shown to act through p300/CBP inhibition, and heterozygous CBP knockout mice are noticeably lean with increased insulin sensitivity (He et al. 2009). The Rt109 HAT was also reported to be required for the pathogenesis of Candida albicans, the most prevalent cause of hospital-acquired fungal infections (Lopes da Rosa et al. 2010). Studies in drug addiction and the related disease of depression using animal models have also uncovered interesting correlations between stages of drug ad- 
diction and histone acetylation status (Renthal and Nestler 2009).

Because of the correlations between several human diseases and histone acetylation balance, proteins that mediate histone acetylation have become attractive drug targets. Although subnanomolar HDAC inhibitors are available, they show poor selectivity among the class I, II, and IV HDACs (Marsoni et al. 2008), and although potent sirtuin activators and inhibitors have been reported they also show modest selectivity (Sanders et al. 2009). HDAC-specific inhibitors are thus actively being pursued, but obtaining them may be particularly challenging because the class I, II, IV, and class III HDACs each share a highly homologous active site and catalytic mechanism (Marsoni et al. 2008).

The development of potent HAT inhibitors has not progressed nearly as far as the development of HDAC inhibitors. The most potent and specific inhibitors have come from the development of peptide-based bisubstrate inhibitors in which coenzyme $\mathrm{A}$ is directly linked to the $\mathrm{N} \zeta$ nitrogen of the target lysine within histone peptides (Lau et al. 2000). Using this technology, Cole and coworkers developed submicromolar inhibitors that show selectivity between the Gcn5/PCAF and p300/CBP HATs (Lau et al. 2000). These bisubstrate inhibitors are a proof of principle that selective HAT inhibitors can be prepared, although peptide-based inhibitors do not generally have favorable pharmacokinetic properties (Heery and Fischer 2007). Nonetheless, structures of such bisubstrate inhibitors have been determined bound to Gcn5 (Poux et al. 2002) and p300 (Figs. 1D and 3B) (Liu et al. 2008). This has provided us with the knowledge of a molecular scaffold from which small molecule compounds with improved pharmacologic properties can be screened against (Hodawadekar and Marmorstein 2007; Wang et al. 2008).

Several natural product HAT inhibitors have been reported including the PCAF and p300 inhibitors, anacardic acid (Fig. 5A) (Sung et al. 2008), and garcinol (Fig. 5B) (Balasubramanyam et al. 2004a). Natural products that have been shown to inhibit p300 include curcumin (Fig. 5C) (Balasubramanyam et al. 2004b), Epigallocatechin-3-
A

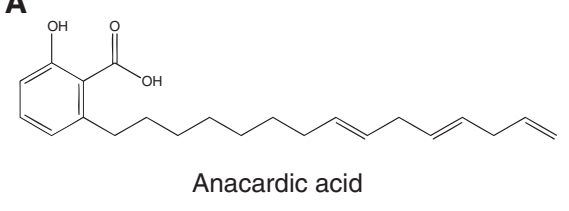

C<smiles>COc1cc(/C=C/C(=O)CC(=O)/C=C/c2ccc(O)c(OC)c2)ccc1O</smiles>

D<smiles>O=C(O[C@H]1Cc2c(O)cc(O)cc2O[C@H]1c1cc(O)c(O)c(O)c1)c1cc(O)c(O)c(O)c1</smiles>

G

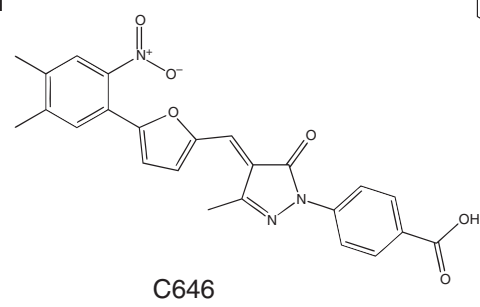

B

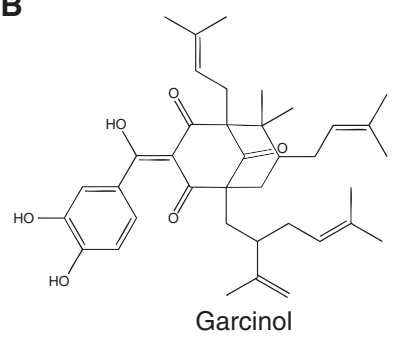

$\mathbf{F}$

E<smiles>CC1=CC(=O)c2c(O)cccc2C(=O)C1=O</smiles><smiles>O=c1cc(Cl)sn1-c1ccc([N+](=O)[O-])cc1</smiles><smiles>COc1ccc(C2CC(c3ccco3)CN2C2=NC(c3ccc(C4C(=O)c5ccccc5C4=O)cc3)CC2)cc1OC</smiles>

I

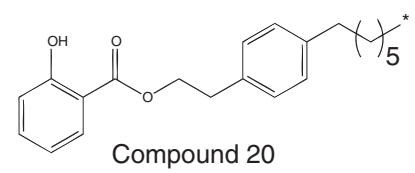

Figure 5. HAT inhibitors. Reported inhibitor specificities are as follows: $(A, B, F)$ specific to p300 and PCAF; $(C-E)$ specific to p300; $(G)$ specific to p300; $(H, I)$ specific to MYST. 
gallate (Fig. 5D) (Choi et al. 2009), and plumbagin (Fig. 5E) (Ravindra et al. 2009). Each of these inhibitors shows $\mathrm{IC}_{50}$ values in the low micromolar range. Although several derivatives of many of these inhibitors have been prepared and evaluated in cells (Heery and Fischer 2007; Furdas et al. 2012), they have not been rigorously biochemically evaluated in vitro and their structures bound to HAT proteins have not been determined, so their mode of action is still unclear.

Some high-throughput screens have been reported, identifying HAT inhibitors with similarly modest potencies to the compounds discussed in the previous paragraph. The high-throughput screening of a 70,000 compound library yielded a family of isothiazolones (CCT077791 and CCT077792) as PCAF inhibitors that were subsequently derivatized as low micromolar PCAF and p300 inhibitors (Fig. 5F) (Gorsuch et al. 2009). Cole and coworkers also used virtual ligand screening of about 50,000 compounds to identify a pyrazolone-containing small molecule p300 HAT inhibitor with $K_{\mathrm{i}} \sim 400 \mathrm{~nm}$ and selectivity against the Gcn5/PCAF, MYST, and Rtt109 HATs (Fig. 5G) (Bowers et al. 2010). More recently, there have been reports of small molecule inhibitors to MYST proteins. Zheng and coworkers performed an in silico inhibitor screen against the AcCoA-binding site of yEsal and then assayed the hits against the yEsa1 and hTIP60 MYST proteins, hp300 and yGcn5 (Wu et al. 2011). This resulted in the identification of four inhibitors that have $\mathrm{IC}_{50}$ values for hTIP60 ranging from $149 \mu \mathrm{M}$ to $400 \mu \mathrm{M}$, with the most potent compound (compound a, Fig. $5 \mathrm{H}$ ) shown to be competitive with AcCoA binding and with some selectivity over Gcn5, but not p300. Zheng and coworkers (Ghizzoni et al. 2012) prepared analogs of the known natural product HAT inhibitor, anacardic acid, and evaluated them as HAT inhibitors. They identified a series of 6-alkylsalicylates that showed selectivity for MYST proteins over Gen 5 and p300, with the most potent compound (20) competitively binding to AcCoA, with $\mathrm{IC}_{50}$ values of $74 \mu \mathrm{M}$ and $47 \mu \mathrm{M}$ for hTIP60 and hMOF, respectively (Fig. 5I). In summary, the HAT inhibitors that have been developed to date only have moderate potency and specificity, therefore motivating the need to develop more potent and selective HAT inhibitors.

\subsection{Conclusions and Open Questions}

Over the last decade, several HAT structures have been determined and their catalytic mechanisms have been characterized. These studies have revealed that HATs fall into distinct subfamilies that show remarkable diversity in amino acid sequence, but retain a structurally conserved core region that mediates AcCoA cofactor and substrate lysine binding. The structurally distinct flanking regions mediate different HAT-associated functions such as histone substrate binding and nucleosome targeting. Remarkably, the different HAT subfamilies use different chemical strategies to acetylate their substrates.

There is much that we still do not understand about HATs, setting the stage for future studies. Open questions include the following: (1) What are the exact molecular details of how autoacetylation regulates HAT function, and is autoacetylation indeed regulatory? It is clear that many HATs, including the p300/CBP, MYST, and Rtt109 subfamilies, are autoacetylated. We currently only have molecular insights into the mode of autoacetylation in MYST proteins. (2) How do associated HAT subunits in multiprotein complexes stimulate, modulate, or coordinate with HAT activity? The structure determination of relevant HAT complexes may address this. (3) What is the molecular basis of HAT substrate specificity? Different HATs appear to harbor different substrate preferences. For example, although the Gcn5/PCAF and Rtt109 HATs have a relatively restricted substrate preference, p300/CBP is considerably more promiscuous. The highly acidic substrate-binding site of p300/CBP has provided some insights into the promiscuity of this HAT subfamily, yet the molecular basis of substrate selectively of other HATs is poorly understood. (4) HAT inhibitors that have been identified to date have relatively modest potency and selectivity profiles. There is clearly significant interest in developing potent and selective small molecule HAT inhibitors. It is likely the structure determination of HAT/inhibitor complexes will be required to facilitate the development of more potent and selective inhibitors with possible therapeutic applications. (5) How much nonhistone acetyltransferases are similar or different to HATs is not currently known, yet is an area of significant interest. Recent proteomic studies on prokaryotic and eukaryotic cells have revealed that protein acetylation reaches beyond histones and transcription-associated biological processes (Choudhary et al. 2009; Zhang et al. 2009). In both eukaryotes and prokaryotes, thousands of acetylated sites have been identified, and protein acetylation occurs in cellular compartments outside of the nucleus, associated with most cellular processes in living organisms including protein translation, protein folding, DNA packaging, and mitochondrial metabolism (Smith and Workman 2009; Spange et al. 2009). Some of the proteins that mediate nonnuclear activities are just beginning to be identified (Ivanov et al. 2002; Akella et al. 2010), but it is likely that there are many other acetyltransferases, with sequence divergence from the currently known histone acetyltransferases, that are yet to be identified. (6) Zhao and coworkers have reported that lysine propionylation and butyrylation occur in cells and these reactions can be catalyzed by the p300 HAT (Chen et al. 2007). Whether known HATs or 
other acetyltransferases can indeed use propionyl-CoA and butyryl-CoA to catalyze such reactions, or whether they are catalyzed by other enzymes in cells to regulate distinct biological processes remains an open question.

\section{ACETYLLYSINE READERS}

\subsection{Structure and Acetyllysine Recognition by Bromodomains}

The bromodomain $(\mathrm{BrD})$ is regarded as the first histonebinding module, whose function is to recognize or "read" acetylated lysine (Dhalluin et al. 1999; Sanchez and Zhou 2009). The bromodomain adopts a distinct structural fold involving a left-handed four-helix bundle $\left(\alpha_{Z}, \alpha_{A}, \alpha_{B}\right.$, and $\alpha_{C}$ ), termed the "BrD fold." The interhelical $\alpha_{Z^{-}} \alpha_{A}$ (ZA) and $\alpha_{B}-\alpha_{C}(B C)$ loops constitute a hydrophobic pocket that recognizes the acetylated lysine (Fig. 6A). Two conserved tyrosine residues in the interhelical loops (one in the ZA loop, the other at the carboxyl terminus of $\alpha_{B}$ ) that contribute to the hydrophobic pocket are found in the majority of bromodomains (Sanchez and Zhou 2009). They are, however, not necessarily the determinants for acetyllysine recognition (Charlop-Powers et al. 2010). A highly conserved asparagine residue at the beginning of the $\mathrm{BC}$ loop (immediately following the second conserved tyrosine) forms a hydrogen bond with the acetyllysine carbonyl oxygen via its side-chain amide nitrogen (Fig. 6B). Together, these characteristics are critical for acetyllysine recognition. Bromodomain binding to acetyllysine in isolation is specific, but occurs with moderate affinity, with dissociation constants $\left(K_{\mathrm{d}}\right)$ typically in the range of tens-to-hundreds micromolar (Vandemark et al. 2007; Zhang et al. 2010).

The overall three-dimensional structure of the bromodomain is well-conserved (Dhalluin et al. 1999; Sanchez and Zhou 2009, and references therein), and changes little on acetyllysine peptide binding, with the exception of the conformational adjustment of the ZA and BC loops. Specificity by the bromodomains is dictated by the sequences within these loops interacting with both the acetylated lysine and up to three or more residues flanking either side of the acetylated lysine (Fig. 6A) (Zeng et al. 2008a; Zhang et al. 2010). The acetylated lysine ligand inserts into the pocket in a similar way as shown in different BrD structures, whether the acetylated lysine-containing ligand is a peptide derived from a histone tail, HIV-1 Tat, or p53 (Mujtaba et al. 2002; Mujtaba et al. 2004).

Bromodomains, like other histone-recognition modules, occur in multiples. The structure of the tandem bromodomains of TAF1 $\left(\mathrm{TAF}_{\mathrm{II}} 250\right)$ shows two bromodomains packed together to form a "U" shape (Fig. 6C) (Jacobson et al. 2000). The individual domains fold independently
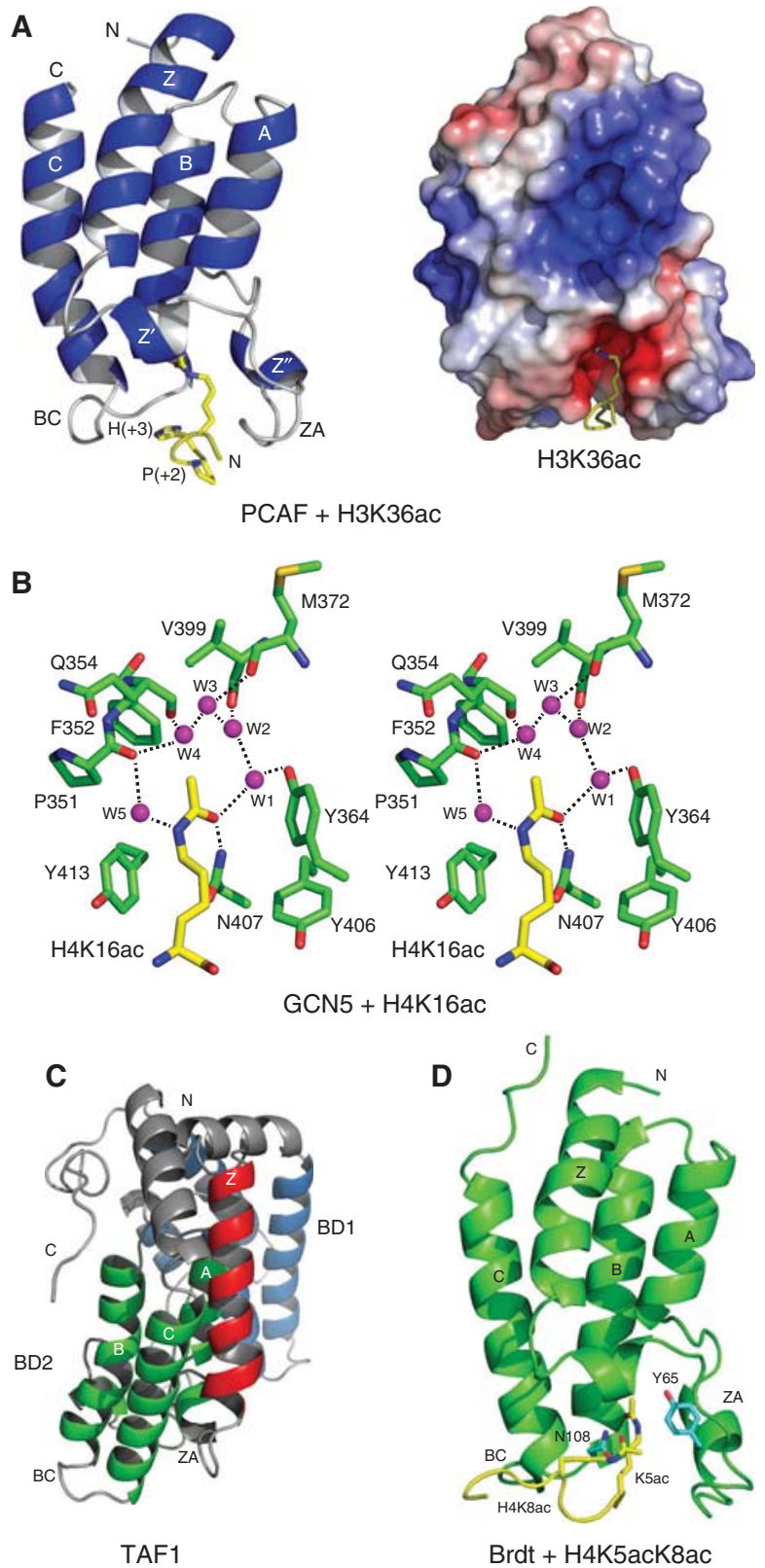

Figure 6. Bromodomains as acetyllysine-binding domains. In all structures, the histone peptide is in yellow and the main and side chains of the protein residues are color-coded by atom type. $(A)$ The three-dimensional solution structure of the PCAF bromodomain bound to an $\mathrm{H} 3 \mathrm{~K} 36 \mathrm{ac}$ peptide (PDB code: $2 \mathrm{RNX}$ ) is illustrated as a ribbon diagram (left) and a surface electrostatic representation (right) of the protein with red and blue colors representing negatively or positively charged amino acid residues, respectively. $(B)$ The acetyllysine-binding pocket is depicted from the crystal structure of the GCN5 bromodomain (green) in complex with an H4K16ac peptide (PDB code: 1E6I). This stick diagram shows key residues and bound water molecules (magenta spheres) contributing to acetyllysine recognition. Hydrogen bonding interactions are indicated by dotted lines. $(C)$ The crystal structure of the tandem bromodomains of uncomplexed human TAF1 (PDB code: 1EQF). (D) The crystal structure of the first bromodomain of Brdt bound to an H4K5acK8ac peptide (PDB code: $2 \mathrm{WP} 2)$. 
and their acetyllysine-binding pockets are $\sim 25 \AA$ apart, equivalent to a span of $7-8$ residues on a peptide. The TAF1 dual bromodomains bind with significantly higher affinity to peptides that are di- or tetra-acetylated at K5/ K12, K8/K16, K5/K8/K12/K16 over a monoacetylated H4 peptide. This is consistent with the notion that each bromodomain binds one acetylated lysine on the same peptide. With the complex structure still elusive, however, this model remains to be confirmed.

A structural study of the tandem bromodomains in yeast Rsc4 showed that only the second bromodomain interacts with an acetylated H3K14 peptide, and this interaction is disrupted by phosphorylation at H3S10 (Vandemark et al. 2007). The two bromodomains fold like one autonomous unit with extensive contacts between the two bromodomains, and are more compact than the TAF1 structure, with the acetyllysine-binding sites $20 \AA$ apart. Further, when a fused $\mathrm{H} 3$ peptide-Rsc 4 dual bromodomain protein was acetylated by $\mathrm{Gcn} 5$, it was found that the amino terminus of Rsc4 was acetylated at a Gcn5-target consensus sequence. Acetylation of the Rsc4 amino-terminal sequence resulted in its binding to the first bromodomain, which precluded binding of the fused histone peptide to the second bromodomain. This suggests that Gen5 provides an autoregulatory mechanism to control Rsc4 activity by acetylating both activating (i.e., H3 lysine acetylation) and inhibiting modifications (e.g., amino-terminal region of Rsc4).

The Polybromo (PB1) protein contains six bromodomains in tandem at its amino terminus and is involved in chromatin remodeling. It was hypothesized that the presence of multiple bromodomains might enable it to recognize specific nucleosomal acetylation patterns that would target PB1 and its parent PBAF complex to chromatin (Thompson 2009). Structures available for all but the fourth bromodomain confirm that secondary and tertiary structure is generally well-conserved among the bromodomains. All but the sixth bromodomain contain an additional two small helices within the ZA loop. However, sequence analysis indicates that these domains may be classified separately from one another, suggesting different ligands and affinities for each (Sanchez and Zhou 2009). Indeed, each bromodomain appears to have preferences for different acetylated lysines on $\mathrm{H} 2 \mathrm{~A}, \mathrm{H} 2 \mathrm{~B}, \mathrm{H} 3$, or H4 (Thompson 2009; Charlop-Powers et al. 2010) although the fifth and sixth bromodomains may serve as nonspecific-binding modules that stabilize PB1 binding to a specific acetylated histone sequence via the other four bromodomains. Notably, in the only PB1 bromodomain complex structure available, the H3K14ac peptide does not interact with the conserved Tyr residues that contribute to the hydrophobic pocket, but instead interacts with Leu and Val residues from the first additional helix within loop ZA and the amino terminus of $\alpha_{C}$, respectively (Charlop-Powers et al. 2010). Because these residues are not conserved among other bromodomains within PB1, it is likely that PB1 uses multiple modes of recognition of their biological ligands.

Some bromodomains have been reported to interact with histones containing more than one acetylated lysine. For example, Brdt, a testis-specific bromodomain and extraterminal domain (BET) protein, recognizes and compacts hyperacetylated chromatin. Brdt contains two tandem bromodomains, the overall structures of which are similar, and their ligand-binding pockets are comparably large. Interestingly, however, the first bromodomain recognizes two acetylated lysines within its pocket (H4K5ac/ $\mathrm{K} 8 \mathrm{ac}$ ), whereas the second recognizes a single acetylated lysine (H3K18ac; Fig. 6D) (Moriniere et al. 2009). Notably, H4K5ac recognition represents the canonical mode of acetyllysine binding by the bromodomain (i.e., interacting with the conserved Asn108 and Tyr65 in Brdt), whereas the acetylated side chain of H4K8ac is largely bound in a hydrophobic cavity on the protein surface consisting of the side chains of residues Trp49, Pro50, Leu60, and Ile114. Despite the general structural similarity of the two bromodomains in Brdt, the sequences in the $\mathrm{ZA}$ and $\mathrm{BC}$ loops differ and determine that only the first bromodomain is able to interact with the $\mathrm{H} 4 \mathrm{~K} 5 \mathrm{ac} / \mathrm{K} 8 \mathrm{ac}$ peptide. Based on sequence conservation, the ligand-binding specificity of the two bromodomains in BET proteins is likely conserved for other BET proteins. Indeed, this mode of one domain interacting with two modifications within a single-binding pocket was also reported recently for BRD4 (Filippakopoulos et al. 2012), and also for BRD3, whose first bromodomain interacts with double acetylated lysines of a nonhistone protein - the hematopoietic transcription factor GATA1 - that is important for erythroid target gene activation (Gamsjaeger et al. 2011; Lamonica et al. 2011).

\subsection{The Human Bromodomain Proteins}

The human genome encodes 42 bromodomain-containing proteins that contain a total of 56 unique individual bromodomains (Schultz et al. 2000; Sanchez and Zhou 2009). The diversity of the human bromodomain family can be illustrated by clustering the 56 bromodomain sequences into eight groups, each of which share similar sequence length and at least 30\% sequence identity (Fig. 7) (Sanchez et al. 2000). Bromodomain proteins represent a wide variety of functionality in chromatin biology and gene transcription. Among the most notable bromodomain proteins are the HATs discussed in Section 2-PCAF, GCN5, and p300/CBP-which function as transcription coactivators. 


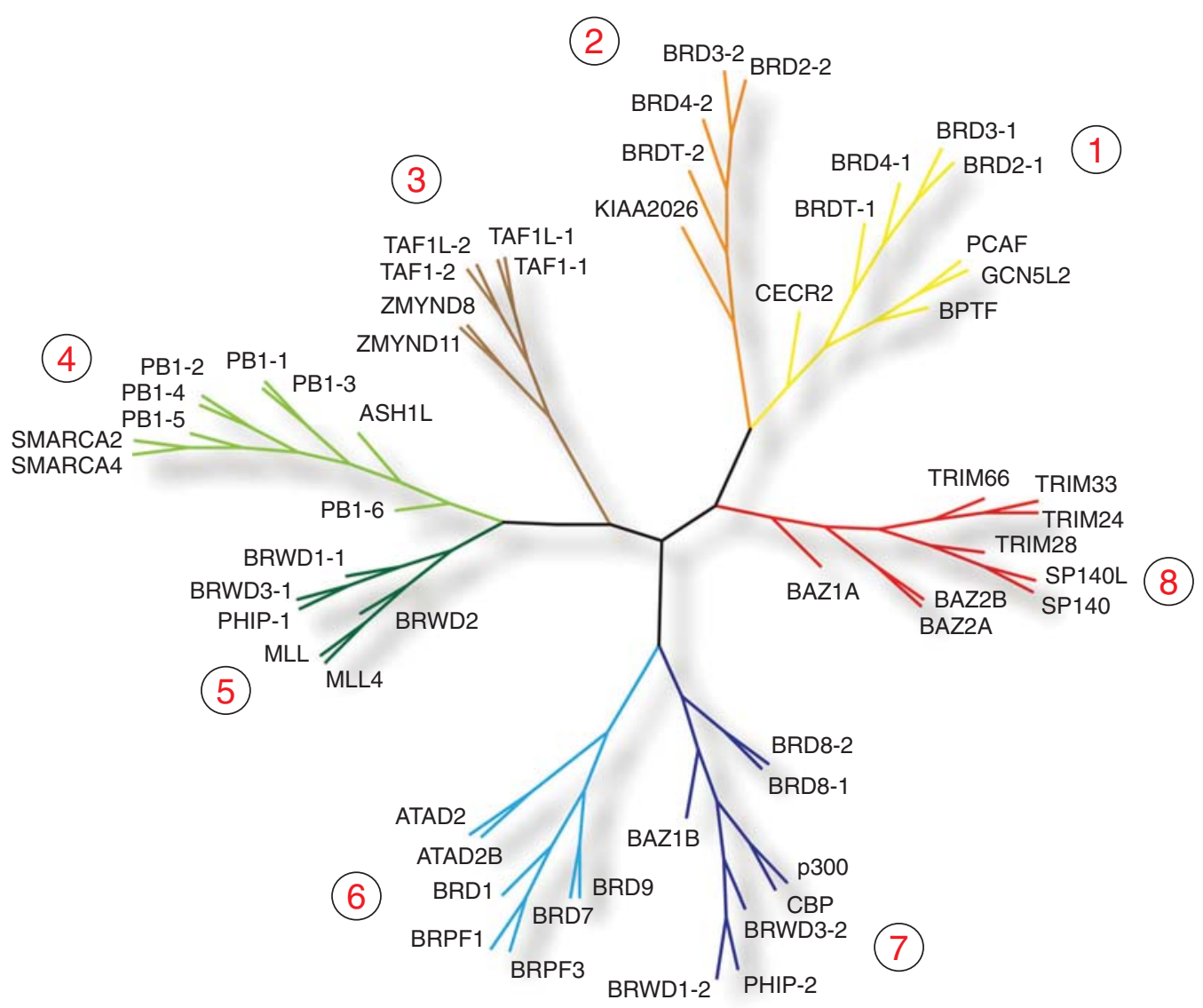

Figure 7. The phylogenetic tree of human bromodomains. Sequence similarity-based dendrogram of the human bromodomains was generated using the neighbor-joining method with MEGA (Kumar et al. 2004). Sequences of the human bromodomains were obtained from the SMART database (Letunic et al. 2004) and aligned with SMART bromodomains' hidden Markov models using Hmmalign (Sonnhammer et al. 1997). (Modified from Zhang et al. 2010.)

It has been suggested that the bromodomains in these nuclear HATs contribute to substrate recruitment and specificity involving histones and nonhistone proteins, thereby providing a functional link between lysine acetylation and acetylation-mediated protein-protein interactions in chromatin-mediated gene transcription (Sanchez and Zhou 2009). Bromodomains are also found in some histone lysine methyltransferases such as ASH1L and MLL, however, a detailed understanding of their function remains elusive.

Bromodomain proteins are involved in chromatin remodeling, the topic of Becker and Workman (2013). Bromodomain-containing remodelers include SMARC2 (also known as BRM, SNF2/SWI2) and SMARC4 (BRG1), and some with ATP-dependent helicase activity include ATAD2 (ATPase family AAA domain-containing protein 2; ANCCA) and ATAD2B. Further, double bromodomains are seen in many proteins including TAF1/TAF1L, the TFIID $250-\mathrm{kDa}$ subunit of the transcription initiation complex, as well as the BET proteins of BRD2, BRD3, BRD4, and the testis-specific protein BRDT. The BET proteins play an important role in the assembly of the productive transcriptional activation complex through the recruitment of the p-TEFb complex (CDK9 and cyclin T1) to RNA polyermase II, which is required for transcriptional elongation (Chiang 2009).

\subsection{The Association of Bromodomains with Other Chromatin Modules}

Bromodomains are often present with a variety of other conserved protein modules of different functions within the same proteins (Basu et al. 2008; Basu et al. 2009). For instance, PCAF, GCN5, p300/CBP are histone lysine acetyltransferases, whereas HRX/ALL-1 is a histone lysine $\mathrm{N}$-methyltransferase and SNF2L2 is an ATP-dependent helicase. More than 15 different domain types have been identified to occur within the same proteins as bromodo- 
mains, including the PHD, PWWP, B-box type zinc finger, ring finger, SAND, FY Rich, SET, TAZ zinc finger, helicase, ATPase, BAH (bromo-adjacent homolog) domain, WD40 repeat, and MBD (methyl-CpG-binding domain) (Schultz et al. 2000).

The modular domain that is most frequently associated with the bromodomain is the PHD (plant homeodomain) finger, which is a $\mathrm{C} 4 \mathrm{HC} 3$ zinc-finger-like motif present in nuclear proteins (Sanchez and Zhou 2011). A PHD has been identified in 19 of the 42 human bromodomain-containing proteins. In 12 of these proteins the PHD and bromodomain are separated by a short amino acid sequence (less than 30 residues). The relative arrangement of the tandem domains vary based on the length and composition of the linker sequence, as well as the residues of the domains that may form an interacting surface. The PHD- bromodomain fragment of KAP1 (also known as TIF1 $\beta$ or TRIM28), a transcriptional corepressor for KRAB zincfinger proteins (Zeng et al. 2008a), lacks several conserved residues within its bromodomain, believed to preclude its ability to directly bind to acetylated lysine. The tandem domains function cooperatively as a single unit, with helix $\alpha_{Z}$ forming a hydrophobic core between the two folds (Fig. 8A) (Zeng et al. 2008b). The intimate association between the domains enables the PHD finger, which is an intramolecular E3 sumoylation ligase, to sumoylate the bromodomain, which enables KAP1 to recruit SETDB1 (a histone H3 lysine 9 specific methyltransferase) to chromatin for gene silencing (Ivanov et al. 2007; Zeng et al. 2008a). Conversely, the PHD-bromodomain tandem module of BPTF, a subunit of a nucleosome-remodeling factor complex, represents two folded domains separated by a helical linker that
A

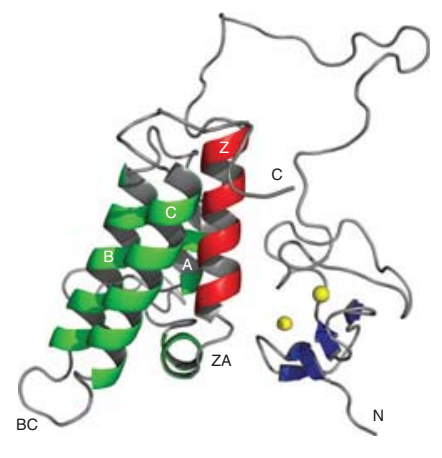

KAP1

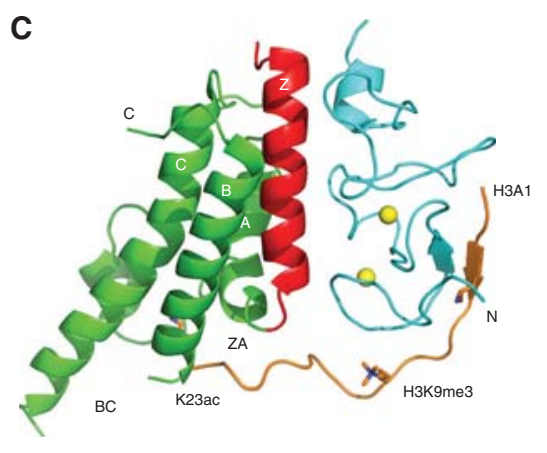

TRIM33 + H3K9me3K18acK23ac
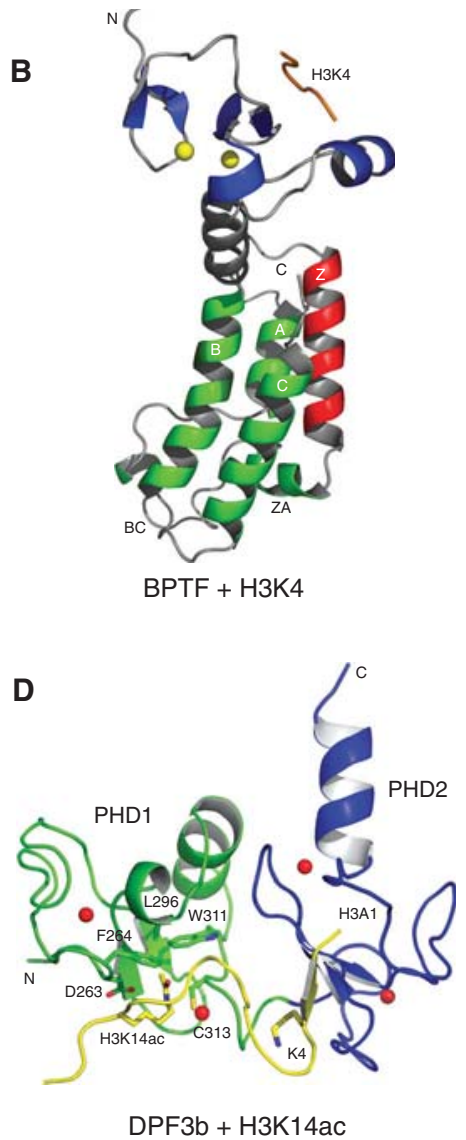

Figure 8. Interdomain interactions in tandem histone-binding modules. (A) The solution structure of the PHD (navy)-bromodomain (red and green) module of human KAP1 (PDB code: 2RO1). (B) The PHD-bromodomain module of human BPTF in complex with an H3K4 peptide (PDB code: 3QZV). (C) The crystal structure of the PHD-bromodomain module of human TRIM33 in complex with an H3K9me3K18acK23ac peptide (PDB code: $3 \mathrm{U} 5 \mathrm{P})$. Note that the second bromodomain in each of the above tandem modules are colored green, and each structure is oriented with respect to the $\alpha_{Z}$ helix (red) of this bromodomain. (D) The solution structure of the tandem PHD finger module of human DPF3b bound to an H3K14ac peptide (PDB code: $2 \mathrm{KWJ}$ ). The zinc atoms are highlighted as red spheres, and the main and side chains of the protein residues involved in H3K14ac binding are color-coded by atom type with green, red, and blue for carbon, oxygen and nitrogen, respectively. 
form no contacts between each other. Transcription proteins such as BPTF (Fig. 8B) (Li et al. 2006), MLL1 (Wang et al. 2010), TRIM24 (Tsai et al. 2010), or TRIM33 (Fig. 8C) (Xi et al. 2011) have tandem PHD domain modules. Several recent studies have shown that these are able to interact with histone $\mathrm{H} 3$ in both acetylation-dependent and methylation-sensitive manner, positively or negatively, thus highlighting a functional coordination between these two important histone modifications in gene transcriptional regulation.

The second most common domain associated with the bromodomain is another bromodomain; 11 of the 42 BRD-containing proteins contain two bromodomains. Polybromo is an exception, containing six bromodomains. In the transcription initiation factors TAF1 and the TFIID $210-\mathrm{kDa}$ subunit, as well as in some of the Polybromo bromodomain pairs, the two bromodomains are separated by short amino acid sequences (less than 20 residues). The structure of the TAF1 bromodomains suggests that they form a tandem arrangement that binds selectively to multiple acetylated histone $\mathrm{H} 4$ peptides (Jacobson et al. 2000).

\subsection{Functions of Human Bromodomain Proteins in Gene Expression}

The complexity and variability of the domain composition in human bromodomain proteins and the influence of neighboring domains (such as the PHD finger) on the function of the bromodomain itself make it difficult to predict functions of bromodomain proteins based on sequence similarity alone. Indeed, growing evidence shows that in addition to histones, bromodomain-containing proteins bind acetylated lysine residues in nonhistone proteins that play an important role in control of gene transcription in chromatin. Many of the human bromodomain proteins do not have well-characterized functions, although some have been implicated in disease processes.

The BET protein BRD4 plays an important role in various biological processes, many of them nonchromatinrelated, by means of its two bromodomains. BRD4 functions in the inflammatory response through interactions with both histone and nonhistone targets. It acts as a coactivator for the transcriptional activation of NF- $\kappa \mathrm{B}$ via the binding of the bromodomains to the acetylated Lys310 on the RelA subunit of NF-кB (Huang et al. 2009). BRD4 also plays a cellular role by stimulating $G_{1}$ gene transcription and promoting cell-cycle progression to the $\mathrm{S}$ phase (Mochizuki et al. 2008). With regard to its role in chromatinrelated processes, a recent study has reported that BRD4 binds, via its bromodomains, to $\mathrm{H} 4 \mathrm{~K} 5 \mathrm{ac}$, and is able to accelerate the dynamics of messenger RNA synthesis by decompacting chromatin and, hence, facilitating transcriptional reactivation (Zhao et al. 2011).

BRD4 can control the transcription of viral genes. For example, this protein has been shown to regulate HIV-1 transcription by inducing the phosphorylation of CDK9 (cyclin-dependent kinase 9) at Thr29 in the HIV transcription initiation complex, thereby inhibiting CDK9 kinase activity and leading to the inhibition of HIV transcription (Zhou et al. 2009). BRD4 is also involved in the inhibition of the proteasomal degradation of the papillomavirus E2 protein (Gagnon et al. 2009). Further, BRD4 associates with Kaposi's sarcoma-associated herpesvirus-encoded LANA-1 (latency-associated nuclear antigen) through molecular interactions involving the carboxy-terminal region (Ottinger et al. 2006) and the extraterminal domain (Lin et al. 2008) of BRD4. Additionally, both BRD4 and BRD2 proteins interact with the murine $\gamma$-herpesvirus 68 protein orf73, which is required to establish viral latency in vivo (Ottinger et al. 2009).

BRD4 plays a role in cancer as well. Its activation may predict the survival of patients with breast cancer (LeRoy et al. 2008). Crawford et al. proposed that the activation of BRD4 manipulates the response to the tumor microenvironment in vivo, resulting in a reduction of tumor growth and pulmonary metastasis in mice (Crawford et al. 2008). Microarray analysis of multiple human mammary tumor cell lines showed that the activation of BRD4 was predictive of a slower rate of cancer progression and/or improved survival. These results suggest that the dysregulation of BRD4-associated pathways likely plays a key role in breast cancer progression.

The coupling of histone acetylation to transcription in vivo by BRD2 and BRD 3 was shown; in human 293 cells, these proteins preferentially associate with specific $\mathrm{H} 4$ modifications along the entire lengths of genes, allowing RNA polymerase II to transcribe through the nucleosomes (LeRoy et al. 2008). In addition, BRD2 also shows histone chaperone activity (LeRoy et al. 2008). BRD2 is essential for murine embryonic development (Shang et al. 2009) and is associated with juvenile myoclonic epilepsy in humans (Pal et al. 2003).

In mice, the bromodomain and WD-repeat-containing protein BRWD1 is required for normal spermiogenesis and the oocyte-embryo transition (Philipps et al. 2008). A mutation in BRWD1 leads to phenotypically normal, but infertile mice. The bromodomain of the HAT transcriptional coactivator $\mathrm{p} 300$ has been suggested to play a role in the IL-6 signaling pathway by mediating the interaction of the STAT3 amide-terminal domain with p300, thereby stabilizing enhanceosome assembly (Hou et al. 2008).

ATAD2, another bromodomain-containing protein, functions as an estrogen-regulated ATPase coactivator in 
both estrogen receptor $\alpha$ and androgen receptor signaling. This protein is required for the formation of transcriptional coregulator complexes in gene expression in chromatin (Zou et al. 2007). Chen and colleagues have suggested that ATAD2 also plays an important role in the development of prostate cancer by mediating specific androgen receptor functions during both cancer cell survival and proliferation (Zou et al. 2009).

\subsection{Pharmacological Inhibition of Bromodomains in Gene Transcription}

Owing to the functional importance of bromodomaincontaining proteins in human biology, chemical modulation of bromodomain/acetyllysine binding, involved in chromatin-mediated gene transcription, is an attractive therapeutic strategy for a number of human diseases (Mujtaba et al. 2006; Prinjha et al. 2012). This strategy was first suggested for controlling HIV-1 transcriptional activation and replication in infected host cells (Zeng et al. 2005). Transcriptional activation of the integrated HIV provirus requires a molecular interaction between the HIV-1 Tat trans-activator, acetylated at lysine 50 and the bromodomain of the host transcriptional coactivator PCAF (Dorr et al. 2002; Mujtaba et al. 2002), suggesting that blocking this host-virus interaction could result in a reduction in Tat-mediated viral transcription. Indeed, small-molecule bromodomain inhibitors such as N1-aryl-propane-1,3-diamine compounds (Fig. 9A) were shown to be able to block PCAF bromodomain binding to K50-acetylated HIV-1 Tat in cells and effectively attenuate Tat-mediated HIV transcriptional activation (Zeng et al. 2005; Pan et al. 2007).
These findings suggest that as a new antiviral strategy for intervening HIV-1 replication, targeting a host cell protein essential for viral reproduction rather than a viral protein may minimize the problem of drug resistance caused by mutations of the viral counterpart as observed with protease inhibitors (Ott et al. 2004).

Mounting evidence shows that many transcription factors undergo site-specific lysine acetylation, and the acetylated lysine then functions to recruit transcription or chromatin effector proteins to facilitate their target gene activation in the chromatin context. One such transcription factor is the human tumor suppressor p53, whose function in gene transcription is dependent on acetylation of several carboxy-terminal lysine residues by HAT coactivators such as CBP. It was shown that lysine acetylation of p53 is important for p53 recruitment of CBP via the CBP bromodomain binding to K382-acetylated p53 for p53 target gene expression (Mujtaba et al. 2004). Studies from the Zhou laboratory have shown that small-molecule chemical- or peptide-based inhibitors, selectively targeting the CBP bromodomain, inhibit p53 transcriptional activity in cells by blocking p53-K382ac binding to the CBP bromodomain, promoting p53 instability by changes in its posttranslational modification states (Mujtaba et al. 2006; Sachchidanand et al. 2006; Gerona-Navarro et al. 2011). Excessive p53 activity has been reported in numerous disease conditions. For instance, during myocardial ischemia, elevated p53 activity causes irreversible cellular injury and cardiomyocyte death. A small molecule named ischemin (Fig. 9A,B) has shown positive effects in reversing the detrimental consequences of myocardial ischemia. This molecule was developed to inhibit the acetyllysine-binding

A

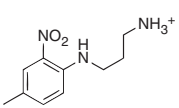

NP1

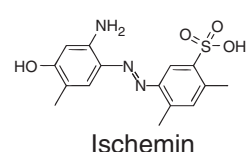

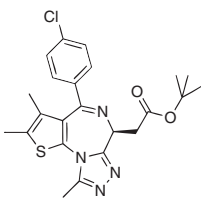

JQ1

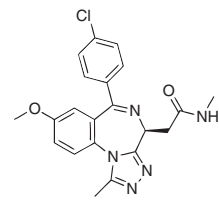

I-BET

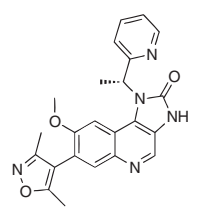

I-BET151
B

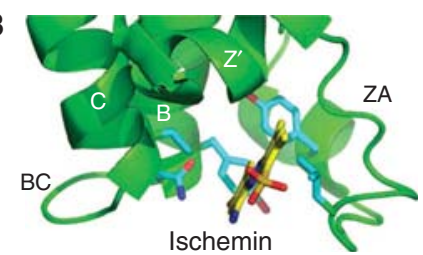

C

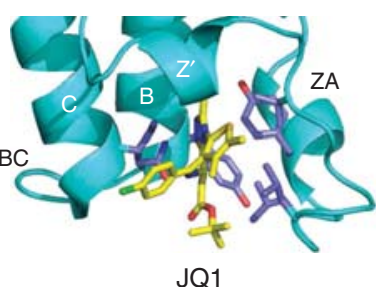

Figure 9. Small molecule inhibitors of bromodomains. $(A)$ Chemical structures of representative small-molecule bromodomain inhibitors, including NP1 (for PCAF bromodomain), ischemin, JQ1, I-BET and I-BET151. (B) Ischemin, a small-molecule inhibitor developed for the CBP bromodomain, depicted in a complexed 3D structure bound to the protein (PDB code: 2L84). (C) JQ1, a BET bromodomain-specific inhibitor, shown when bound to the first bromodomain of BRD4 in the crystal structure (PDB code: 3MXF). 
activity of the bromodomain of CBP. The study further showed that cells treated with ischemin have altered posttranslational modifications on p53 and histones, inhibit the interaction between p53 and CBP, and reduce p53mediated transcriptional activity in cells. Overall, this leads to the prevention of apoptosis in ischemic cardiomyocytes (Borah et al. 2011). These studies suggest that small-molecule modulation of acetylation-mediated interactions in gene transcription can serve as a new approach to therapeutic interventions of human disorders such as myocardial ischemia.

More recently, several highly selective and potent smallmolecule inhibitors for the BET family bromodomain proteins have been developed (Fig. 9A,C) (Prinjha et al. 2012). For instance, the work by Bradner and colleagues first reported that a BET bromodomain-specific inhibitor JQ1 blocks the binding of BRD4 bromodomainsto lysine-acetylated histone H4 (Filippakopoulos et al. 2010). BRD4 is a known component of the recurrent chromosomal translocation product with the NUT protein in an aggressive human squamous carcinoma. Competitive binding by JQ1 displaces the BRD4-NUT fusion oncoprotein from chromatin, prompting squamous differentiation and specific antiproliferative effects in both BRD4-dependent cell lines and patient-derived xenograft models (Filippakopoulos et al. 2010). In another study, Vakoc and coworkers studied acute myeloid leukemia, an aggressive hematopoietic malignancy that is characteristic of altered epigenetic landscapes (Zuber et al. 2011). Suppression of Brd4 by shRNA or inhibition of BRD4 by JQ1 led to dramatic antileukemic effects both in vivo and in vitro, and also led to myeloid differentiation and leukemia stem-cell depletion. Inhibition of BET protein recruitment to chromatin with smallmolecule bromodomain inhibitors including I-BET and IBET151 (Fig. 9A) has also been shown as an effective treatment for MLL-fusion leukemia (Dawson et al. 2011). In their study of multiple myeloma, a Myc oncoproteindependent hematologic malignancy, Delmore et al. have shown that the BET-specific inhibitor JQ1 generates a potent antiproliferative effect associated with cell cycle arrest and cellular senescence (Delmore et al. 2011). These studies suggest that inhibitors of BET bromodomains represent a new therapeutic strategy for malignancies that are characterized by pathologic activation of $c-M y c$ (Delmore et al. 2011; Mertz et al. 2011). Finally, BET bromomdomain inhibition can reduce transcriptional activation of proinflammatory cytokines. For example, I-BET has been shown to be an effective means of down-regulating inflammatory gene expression in activated macrophages, conferring protection against lipopolysaccharide-induced endotoxic shock, and bacteria-induced sepsis (Nicodeme et al. 2010). Moreover, MS417, an improved thienodiazepine-based
BET-specific bromodomain inhibitor (about five- to 10fold more potent than JQ1) can attenuate HIV-infection triggered NF- $\kappa \mathrm{B}$ transcriptional activity in activation of proinflammatory genes in mouse kidney cells, and also ameliorate HIV-induced kidney injury in HIV transgenic mice treated with MS417 (Zhang et al. 2012).

\subsection{Other Acetyllysine Readers}

Acetyllysine binding was regarded a unique molecular function exclusive to the bromodomains following its discovery in 1999 (Dhalluin et al. 1999). In 2008, Lange and coworkers reported that the tandem PHD fingers of human DPF3b, a component of the BAF chromatin remodeling complex, interacts with histone $\mathrm{H} 3$ in a manner sensitive to lysine acetylation and methylation (Lange et al. 2008). The PHD finger is a highly versatile protein module that has been shown to interact with histone $\mathrm{H} 3$ sequences in a modification-sensitive manner (Sanchez and Zhou 2011). A structural analysis of the tandem PHD fingers (PHD12) of human DPF3b provided a detailed molecular basis for the idea that its binding to histone $\mathrm{H} 3$ is modulated positively by lysine acetylation but negatively by lysine methylation (Zeng et al. 2010). Specifically, the tandem PHD fingers fold as one functionally cooperative unit, and interact with an unmodified $\mathrm{H} 3$ peptide with an affinity of $K_{d}$ $\sim 2 \mu \mathrm{M}$; acetylation at K14 enhances $\mathrm{H} 3$ binding to a $K_{d}$ of $0.5 \mu \mathrm{M}$, whereas methylation at $\mathrm{H} 3 \mathrm{~K} 4$ almost abolishes the interaction. The $\mathrm{H} 3$ peptide lies across a surface shared by the two domains with a $\beta$-strand from $\mathrm{R} 2-\mathrm{K} 4$ of $\mathrm{H} 3$ that contributes to the $\beta$-sheet of the second PHD finger (PHD2), a sharp kink at the middle of the peptide due to interactions between the first PHD finger (PHD1) and K9, and K14ac interacting with a hydrophobic pocket formed by PHD1 (Fig. 8D). Additionally, the H3K14ac acyl chain interacts with Arg289 and Phe264 of PHD1, and the acetyl amide group forms a hydrogen bond with the side chain of Asp263 of PHD1. The complex structure of DPF3b PHD12 bound to an amino-terminally acetylated $\mathrm{H} 4$ peptide confirms that the PHD1 interacts with the acetyl group with the same residues. The recognition of the acetyllysine by DPF3b PHD1, however, is structurally distinct from the pattern used by the bromodomains, and is completely different from PHD finger's mechanism for methyl-lysine recognition, which uses a surface on the opposite side of the fold.

\subsection{Conclusions and Open Questions}

The role of the bromodomain as the main protein domain known to recognize acetyllysine residues on proteins involved in gene transcription is much more complex and 
broad than initially envisioned (Dhalluin et al. 1999; Jacobson et al. 2000; Sanchez and Zhou 2009). Despite the large family size of the bromodomains, the acetyllysine recognition mechanism appears strictly conserved. However, it appears that our current knowledge on sequence-dependent recognition by individual bromodomains is very limited. This is in part because of the binding affinity of lysineacetylated histones or nonhistone proteins by bromodomains is quote modest (in the range of tens-to-hundreds micromolar). Furthermore, the high degree of sequence variation and conformational flexibility of bromodomain $\mathrm{ZA}$ and $\mathrm{BC}$ loops, which comprise the acetyllysine-binding pockets, strongly indicates that more structural characterization of bromodomains in complex with their true biological ligands is required. Studies of individual bromodomains have identified varied ligand-binding specificities that are dependent not only on the characteristics of the bromodomain itself, but also on the other protein domains present in the same protein. Studies of bromodomain-containing proteins have highlighted the role of these domains in many important biological processes and their association with disease. The characterization of the multiplicity of molecular interactions mediated by bromodomains is therefore essential for deciphering the role of individual domains and proteins in chromatin-dependent gene transcription. This challenging task may be facilitated by the high structural coverage of the human bromodomain family, which presents a unique opportunity for the rational design of selective small molecules that could serve as tools to modulate and control gene expression in human biology. The more recent observation that the PHD fingers can also bind acetylated lysine residues also leaves open the possibility that still other types of domains may also be used for acetyllysine recognition.

\section{PERSPECTIVES}

Over the last decade we have learned that the writers and readers of lysine acetylation have many fascinating and unanticipated properties. The HAT enzymes that create the modification come in many different flavors, are regulated by autoacetylation, and interact with other protein subunits. They acetylate many different substrates-histone and nonhistone- to mediate diverse biological process and are attractive drug targets because of their association with diseases such as cancers and metabolic disorders. Acetylation marks are also recognized ("read") by dedicated protein modules such as bromodomains, PHD fingers, and possibly other types of domains, to mediate downstream biological signals. Interestingly, many of the properties listed for lysine acetyltransferases are common to the superfamily of kinase enzymes, including the recognition of phospho marks by protein segments such as SH2, PTB, and FHA domains (Taylor and Kornev 2011). However, because studies of kinases are more extensive and date back further, they are much better understood. Indeed, one could argue that the acetylation pathway for signal transduction and gene expression is in the same position phosphorylation was several decades ago. This raises the question: Does acetylation rival phosphorylation for mediating key signal transduction events in biology? Time will tell.

\section{ACKNOWLEDGMENTS}

The work was supported in part by the National Institutes of Health grants GM060293, GM098910, and AG031862 awarded to R.M., and grants CA87658 and HG004508 awarded to M.-M.Z.

\section{REFERENCES}

* Reference is also in this collection.

Ai X, Parthun MR. 2004. The nuclear Hat1p/Hat2p complex: A molecular link between type B histone acetyltransferases and chromatin assembly. Mol Cell 14: 195-205.

Akella JS, Wloga D, Kim J, Starostina NG, Lyons-Abbott S, Morrissette NS, Dougan ST, Kipreos ET, Gaertig J. 2010. MEC-17 is an $\alpha$-tubulin acetyltransferase. Nature 467: 218-222.

Akhtar A, Becker PB. 2001. The histone H4 acetyltransferase MOF uses a $\mathrm{C}_{2} \mathrm{HC}$ zinc finger for substrate recognition. EMBO Rep 2: 113-118.

Albaugh BN, Kolonko EM, Denu JM. 2010. Kinetic mechanism of the Rtt109-Vps75 histone acetyltransferase-chaperone complex. Biochemistry 49: 6375-6385.

Albaugh BN, Arnold KM, Lee S, Denu JM. 2011. Autoacetylation of the histone acetyltransferase RTT109. J Biol Chem 286: 24694-24701.

Allis CD, Berger SL, Cote J, Dent S, Jenuwien T, Kouzarides T, Pillus L, Reinberg D, Shi Y, Shiekhattar R, et al. 2007. New nomenclature for chromatin-modifying enzymes. Cell 131: 633-636.

* Almouzni G, Cedar H. 2014. Maintenance of epigenetic information. Cold Spring Harb Perspect Biol doi: 10.1101/cshperspect.a019372.

Balasubramanyam K, Altaf M, Varier RA, Swaminathan V, Ravindran A, Sadhale PP, Kundu TK. 2004a. Polyisoprenylated benzophenone, garcinol, a natural histone acetyltransferase inhibitor, represses chromatin transcription and alters global gene expression. J Biol Chem 279: 33716-33726.

Balasubramanyam K, Varier RA, Altaf M, Swaminathan V, Siddappa NB, Ranga U, Kundu TK. 2004b. Curcumin, a novel p300/CREB-binding protein-specific inhibitor of acetyltransferase, represses the acetylation of histone/nonhistone proteins and histone acetyltransferase-dependent chromatin transcription. J Biol Chem 279: 51163-51171.

Bannister AJ, Kouzarides T. 2011. Regulation of chromatin by histone modifications. Cell Res 21: 381-395.

Barnes PJ, Adcock IM, Ito K. 2005. Histone acetylation and deacetylation: Importance in inflammatory lung diseases. Eur Respir J 25: 552-563.

Basu MK, Carmel L, Rogozin IB, Koonin EV. 2008. Evolution of protein domain promiscuity in eukaryotes. Genome Res 18: 449-461.

Basu MK, Poliakov E, Rogozin IB. 2009. Domain mobility in proteins: Functional and evolutionary implications. Brief Bioinform 10: 205216.

* Becker PB, Workman JL. 2013. Nucleosome remodeling and epigenetics. Cold Spring Harb Perspect Biol doi: 10.1101/cshperspect.a017905. 
Berndsen CE, Albaugh BN, Tan S, Denu JM. 2007. Catalytic mechanism of a MYST family histone acetyltransferase. Biochemistry 46: 623-629.

Berndsen CE, Tsubota T, Lindner SE, Lee S, Holton JM, Kaufman PD, Keck JL, Denu JM. 2008. Molecular functions of the histone acetyltransferase chaperone complex Rtt109-Vps75. Nat Struct Mol Biol 15: 948-956.

Borah JC, Mujtaba S, Karakikes I, Zeng L, Muller M, Patel J, Moshkina N, Morohashi K, Zhang W, Gerona-Navarro G, et al. 2011. A small molecule binding to the coactivator CREB-binding protein blocks apoptosis in cardiomyocytes. Chem Biol 18: 531-541.

Bowers EM, Yan G, Mukherjee C, Orry A, Wang L, Holbert MA, Crump NT, Hazzalin CA, Liszczak G, Yuan H, et al. 2010. Virtual ligand screening of the p300/CBP histone acetyltransferase: Identification of a selective small molecule inhibitor. Chem Biol 17: 471-482.

Brent MM, Marmorstein R. 2008. Ankyrin for methylated lysines. Nat Struct Mol Biol 15: 221-222.

Brownell JE, Zhou J, Ranalli T, Kobayashi R, Edmondson DG, Roth SY, Allis CD. 1996. Tetrahymena histone acetyltransferase A: A homolog of yeast Gcn5p linking histone acetylation to gene activation. Cell 84: $843-851$.

* Busslinger M, Tarakhovsky A. 2014. Epigenetic control of immunity. Cold Spring Harb Perspect Biol doi: 10.1101/cshperspect.a019307.

Carrozza MJ, Utley RT, Workman JL, Cote J. 2003. The diverse functions of histone acetyltransferase complexes. Trends Genet 19: 321-329.

Cereseto A, Manganaro L, Gutierrez MI, Terreni M, Fittipaldi A, Lusic M, Marcello A, Giacca M. 2005. Acetylation of HIV-1 integrase by p300 regulates viral integration. EMBO J 24: 3070-3081.

Charlop-Powers Z, Zeng L, Zhang Q, Zhou MM. 2010. Structural insights into selective histone $\mathrm{H} 3$ recognition by the human Polybromo bromodomain 2. Cell Res 20: 529-538.

Chen Y, Sprung R, Tang Y, Ball H, Sangras B, Kim SC, Falck JR, Peng J, Gu W, Zhao Y. 2007. Lysine propionylation and butyrylation are novel post-translational modifications in histones. Mol Cell Proteomics 6: $812-819$.

Chiang CM. 2009. Brd4 engagement from chromatin targeting to transcriptional regulation: Selective contact with acetylated histone $\mathrm{H} 3$ and H4. F1000 Biol Rep 1: 98.

Choi KC, Jung MG, Lee YH, Yoon JC, Kwon SH, Kang HB, Kim MJ, Cha JH, Kim YJ, Jun WJ, et al. 2009. Epigallocatechin-3-gallate, a histone acetyltransferase inhibitor, inhibits EBV-induced B lymphocyte transformation via suppression of RelA acetylation. Cancer Res 69: 583592.

Choudhary C, Kumar C, Gnad F, Nielsen ML, Rehman M, Walther TC, Olsen JV, Mann M. 2009. Lysine acetylation targets protein complexes and co-regulates major cellular functions. Science 325: 834-840.

Clements A, Rojas JR, Trievel RC, Wang L, Berger SL, Marmorstein R. 1999. Crystal structure of the histone acetyltransferase domain of the human PCAF transcriptional regulator bound to coenzyme A. EMBO J 18: 3521-3532.

Clements A, Poux AN, Lo WS, Pillus L, Berger SL, Marmorstein R. 2003. Structural basis for histone and phosphohistone binding by the GCN5 histone acetyltransferase. Mol Cell 12: 461-473.

Crawford NP, Alsarraj J, Lukes L, Walker RC, Officewala JS, Yang HH, Lee MP, Ozato K, Hunter KW. 2008. Bromodomain 4 activation predicts breast cancer survival. Proc Natl Acad Sci 105: 6380-6385.

Dawson MA, Prinjha RK, Dittmann A, Giotopoulos G, Bantscheff M, Chan WI, Robson SC, Chung CW, Hopf C, Savitski MM, et al. 2011. Inhibition of BET recruitment to chromatin as an effective treatment for MLL-fusion leukaemia. Nature 478: 529-533.

Delmore JE, Issa GC, Lemieux ME, Rahl PB, Shi J, Jacobs HM, Kastritis E, Gilpatrick T, Paranal RM, Qi J, et al. 2011. BET bromodomain inhibition as a therapeutic strategy to target c-Myc. Cell 146: 904-917.

Dhalluin C, Carlson J, Zeng L, He C, Aggarwal K, Zhou MM. 1999. Structure and ligand of a histone acetyltransferase bromodomain. Nature 399: 491-496.

Doi M, Hirayama J, Sassone-Corsi P. 2006. Circadian regulator CLOCK is a histone acetyltransferase. Cell 125: 497-508.
Dorr A, Kiermer V, Pedal A, Rackwitz H, Henklein P, Schubert U, Zhou M, Verdin E, Ott M. 2002. Transcriptional synergy between Tat and PCAF is dependent on the binding of acetylated Tat to the PCAF bromodomain. EMBO J 21: 2715-2723.

Driscoll R, Hudson A, Jackson SP. 2007. Yeast Rtt109 promotes genome stability by acetylating histone H3 on lysine 56 . Science 315: 649-652.

Dutnall RN, Tafrov ST, Sternglanz R, Ramakrishnan V. 1998. Structure of the histone acetyltransferase Hat1: A paradigm for the GCN5-related $\mathrm{N}$-acetyltransferase superfamily. Cell 94: 427-438.

Filippakopoulos P, Qi J, Picaud S, Shen Y, Smith WB, Fedorov O, Morse EM, Keates T, Hickman TT, Felletar I, et al. 2010. Selective inhibition of BET bromodomains. Nature 468: 1067-1073.

Filippakopoulos P, Picaud S, Mangos M, Keates T, Lambert JP, BarsyteLovejoy D, Felletar I, Volkmer R, Muller S, Pawson T, et al. 2012. Histone recognition and large-scale structural analysis of the human bromodomain family. Cell 149: 214-231.

Furdas SD, Kannan S, Sippl W, Jung M. 2012. Small molecule inhibitors of histone acetyltransferases as epigenetic tools and drug candidates. Arch Pharm (Weinheim) 345: 7-21.

Gagnon D, Joubert S, Senechal H, Fradet-Turcotte A, Torre S, Archambault J. 2009. Proteasomal degradation of the papillomavirus E2 protein is inhibited by overexpression of bromodomain-containing protein 4. J Virol 83: 4127-4139.

Gamsjaeger R, Webb SR, Lamonica JM, Billin A, Blobel GA, Mackay JP. 2011. Structural basis and specificity of acetylated transcription factor GATA1 recognition by BET family bromodomain protein Brd3. Mol Cell Biol 31: 2632-2640.

Gerona-Navarro G, Yoel R, Mujtaba S, Frasca A, Patel J, Zeng L, Plotnikov AN, Osman R, Zhou MM. 2011. Rational design of cyclic peptide modulators of the transcriptional coactivator CBP: A new class of p53 inhibitors. J Am Chem Soc 133: 2040-2043.

Ghizzoni M, Wu J, Gao T, Haisma HJ, Dekker FJ, Zheng YG. 2012. 6alkylsalicylates are selective Tip60 inhibitors and target the acetyl-CoA binding site. Eur J Med Chem 47: 337-344.

Glatt S, Alfieri C, Muller CW. 2011. Recognizing and remodeling the nucleosome. Curr Opin Struct Biol 21: 335-341.

Gorsuch S, Bavetsias V, Rowlands MG, Aherne GW, Workman P, Jarman M, McDonald E. 2009. Synthesis of isothiazol-3-one derivatives as inhibitors of histone acetyltransferases (HATs). Bioorg Med Chem 17: $467-474$

Han J, Zhou H, Li Z, Xu RM, Zhang Z. 2007a. Acetylation of lysine 56 of histone H3 catalyzed by RTT109 and regulated by ASF1 is required for replisome integrity. J Biol Chem 282: 28587-28596.

Han J, Zhou H, Li Z, Xu RM, Zhang Z. 2007b. The Rtt109-Vps75 histone acetyltransferase complex acetylates non-nucleosomal histone $\mathrm{H} 3$. J Biol Chem 282: 14158-14164.

He L, Sabet A, Djedjos S, Miller R, Sun X, Hussain MA, Radovick S, Wondisford FE. 2009. Metformin and insulin suppress hepatic gluconeogenesis through phosphorylation of CREB binding protein. Cell 137: 635-646.

Heery DM, Fischer PM. 2007. Pharmacological targeting of lysine acetyltransferases in human disease: A progress report. Drug Discov Today 12: $88-99$.

Hodawadekar SC, Marmorstein R. 2007. Chemistry of acetyl transfer by histone modifying enzymes: Structure, mechanism and implications for effector design. Oncogene 26: 5528-5540.

Holbert MA, Sikorski T, Carten J, Snowflack D, Hodawadekar S, Marmorstein R. 2007. The human monocytic leukemia zinc finger histone acetyltransferase domain contains DNA-binding activity implicated in chromatin targeting. J Biol Chem 282: 36603-36613.

Hou T, Ray S, Lee C, Brasier AR. 2008. The STAT3 $\mathrm{NH}_{2}$-terminal domain stabilizes enhanceosome assembly by interacting with the p300 bromodomain. J Biol Chem 283: 30725-30734.

Huang B, Yang XD, Zhou MM, Ozato K, Chen LF. 2009. Brd4 coactivates transcriptional activation of NF- $\mathrm{KB}$ via specific binding to acetylated RelA. Mol Cell Biol 29: 1375-1387. 
Ivanov AV, Peng H, Yurchenko V, Yap KL, Negorev DG, Schultz DC, Psulkowski E, Fredericks WJ, White DE, Maul GG, et al. 2007. PHD domain-mediated E3 ligase activity directs intramolecular sumoylation of an adjacent bromodomain required for gene silencing. Mol Cell 28: $823-837$.

Ivanov D, Schleiffer A, Eisenhaber F, Mechtler K, Haering CH, Nasmyth K. 2002. Ecol is a novel acetyltransferase that can acetylate proteins involved in cohesion. Curr Biol 12: 323-328.

Jacobson RH, Ladurner AG, King DS, Tjian R. 2000. Structure and function of a human $\mathrm{TAF}_{\mathrm{II}} 250$ double bromodomain module. Science 288: $1422-1425$.

Karanam B, Wang L, Wang D, Liu X, Marmorstein R, Cotter R, Cole PA. 2007. Multiple roles for acetylation in the interaction of p300 HAT with ATF-2. Biochemistry 46: 8207-8216.

Kawasaki H, Schiltz L, Chiu R, Itakura K, Taira K, Nakatani Y, Yokoyama KK. 2000. ATF-2 has intrinsic histone acetyltransferase activity which is modulated by phosphorylation. Nature 405: 195-200.

Keppler BR, Archer TK. 2008a. Chromatin-modifying enzymes as therapeutic targets-Part 1. Expert Opin Ther Targets 12: 1301-1312.

Keppler BR, Archer TK. 2008b. Chromatin-modifying enzymes as therapeutic targets-Part 2. Expert Opin Ther Targets 12: 1457-1467.

Kleff S, Andrulis ED, Anderson CW, Sternglanz R. 1995. Identification of a gene encoding a yeast histone $\mathrm{H} 4$ acetyltransferase. J Biol Chem 270: 24674-24677.

Kolonko EM, Albaugh BN, Lindner SE, Chen Y, Satyshur KA, Arnold KM, Kaufman PD, Keck JL, Denu JM. 2010. Catalytic activation of histone acetyltransferase Rtt109 by a histone chaperone. Proc Natl Acad Sci 107: 20275-20280.

Kumar S, Tamura K, Nei M. 2004. MEGA3: Integrated software for molecular evolutionary genetics analysis and sequence alignment. Brief Bioinform 5: 150-163.

Kurth HM, Mochizuki K. 2009. Non-coding RNA: A bridge between small RNA and DNA. RNA Biol 6: 138-140.

Lamonica JM, Deng W, Kadauke S, Campbell AE, Gamsjaeger R, Wang H, Cheng Y, Billin AN, Hardison RC, Mackay JP, et al. 2011. Bromodomain protein Brd3 associates with acetylated GATA1 to promote its chromatin occupancy at erythroid target genes. Proc Natl Acad Sci 108: E159-E168.

Lange M, Kaynak B, Forster UB, Tonjes M, Fischer JJ, Grimm C, Schlesinger J, Just S, Dunkel I, Krueger T, et al. 2008. Regulation of muscle development by DPF3, a novel histone acetylation and methylation reader of the BAF chromatin remodeling complex. Genes Dev 22: $2370-2384$.

Lau OD, Kundu TK, Soccio RE, Ait-Si-Ali S, Khalil EM, Vassilev A, Wolffe AP, Nakatani Y, Roeder RG, Cole PA. 2000. HATs off: Selective synthetic inhibitors of the histone acetyltransferases p300 and PCAF. Mol Cell 5: 539-595.

Lee KK, Workman JL. 2007. Histone acetyltransferase complexes: One size doesn't fit all. Nat Rev Mol Cell Biol 8: 284-295.

LeRoy G, Rickards B, Flint SJ. 2008. The double bromodomain proteins Brd2 and Brd3 couple histone acetylation to transcription. Mol Cell 30: $51-60$.

Letunic I, Copley R, Schmidt S, Ciccarelli F, Doerks T, Schultz J, Ponting C, Bork P. 2004. SMART 4.0: Towards genomic data integration. Nucleic Acids Res 32: D142-D144.

Li H, Ilin S, Wang W, Duncan EM, Wysocka J, Allis CD, Patel DJ. 2006. Molecular basis for site-specific read-out of histone H3K4me3 by the BPTF PHD finger of NURF. Nature 442: 91-95.

Lin C, Yuan YA. 2008. Structural insights into histone H3 lysine 56 acetylation by Rtt109. Structure 16: 1503-1510.

Lin Y, Fletcher CM, Zhou J, Allis CD, Wagner G. 1999. Solution structure of the catalytic domain of Tetrahymena GCN5 histone acetyltransferase in complex with coenzyme A. Nature 400: 86-89.

Lin YJ, Umehara T, Inoue M, Saito K, Kigawa T, Jang MK, Ozato K, Yokoyama S, Padmanabhan B, Guntert P. 2008. Solution structure of the extraterminal domain of the bromodomain-containing protein BRD4. Protein Sci 17: 2174-2179.
Liu X, Wang L, Zhao K, Thompson PR, Hwang Y, Marmorstein R, Cole PA. 2008. The structural basis of protein acetylation by the p300/CBP transcriptional coactivator. Nature 451: 846-850.

Lopes da Rosa J, Boyartchuk VL, Zhu LJ, Kaufman PD. 2010. Histone acetyltransferase Rtt109 is required for Candida albicans pathogenesis. Proc Natl Acad Sci 107: 1594-1599.

Lu L, Li L, Lv X, Wu XS, Liu DP, Liang CC. 2011. Modulations of hMOF autoacetylation by SIRT1 regulate hMOF recruitment and activities on the chromatin. Cell Res 21: 1182-1185.

Marmorstein R, Trievel RC. 2009. Histone modifying enzymes: Structures, mechanisms, and specificities. Biochim Biophys Acta 1789: $58-68$.

Marsoni S, Damia G, Camboni G. 2008. A work in progress: The clinical development of histone deacetylase inhibitors. Epigenetics 3: 164-171.

Mattick JS, Makunin IV. 2006. Non-coding RNA. Hum Mol Genet 15: R17-R29.

Mertz JA, Conery AR, Bryant BM, Sandy P, Balasubramanian S, Mele DA, Bergeron L, Sims RJ 3rd. 2011. Targeting MYC dependence in cancer by inhibiting BET bromodomains. Proc Natl Acad Sci 108: 1666916674.

Mizzen CA, Yang X-J, Kokubo T, Brownell JE, Bannister AJ, OwenHughes T, Workman J, Wang L, Berger SL, Kouzarides T, et al. 1996. The TAF $_{\text {II }} 250$ subunit of TFIID has histone acetyltransferase activity. Cell 87: $1261-1270$.

Mochizuki K, Nishiyama A, Jang MK, Dey A, Ghosh A, Tamura T, Natsume $\mathrm{H}$, Yao H, Ozato K. 2008. The bromodomain protein Brd4 stimulates $\mathrm{G}_{1}$ gene transcription and promotes progression to $S$ phase. J Biol Chem 283: 9040-9048.

Moriniere J, Rousseaux S, Steuerwald U, Soler-Lopez M, Curtet S, Vitte AL, Govin J, Gaucher J, Sadoul K, Hart DJ, et al. 2009. Cooperative binding of two acetylation marks on a histone tail by a single bromodomain. Nature 461: 664-668.

Mujtaba S, He Y, Zeng L, Farooq A, Carlson J, Ott M, Verdin E, Zhou M. 2002. Structural basis of lysine-acetylated HIV-1 Tat recognition by PCAF bromodomain. Mol Cell 9: 575-586.

Mujtaba S, He Y, Zeng L, Yan S, Plotnikova O, Sachchidanand, Sanchez R, Zeleznik-Le NJ, Ronai Z, Zhou MM. 2004. Structural mechanism of the bromodomain of the coactivator CBP in p53 transcriptional activation. Mol Cell 13: 251-263.

Mujtaba S, Zeng L, Zhou MM. 2006. Modulating molecular functions of p53 with small molecules. Cell Cycle 5: 2575-2578.

Nagy Z, Tora L. 2007. Distinct GCN5/PCAF-containing complexes function as co-activators and are involved in transcription factor and global histone acetylation. Oncogene 26: 5341-5357.

Neuwald AF, Landsman D. 1997. GCN5-related histone $N$-acetyltransferases belong to a diverse superfamily that include the yeast SPT10 protein. Trends Biochem Sci 22: 154-155.

Nicodeme E, Jeffrey KL, Schaefer U, Beinke S, Dewell S, Chung CW, Chandwani R, Marazzi I, Wilson P, Coste H, et al. 2010. Suppression of inflammation by a synthetic histone mimic. Nature 468: 11191123.

Ott M, Dorr A, Hetzer-Egger C, Kaehlcke K, Schnolzer M, Henklein P, Cole P, Zhou M, Verdin E. 2004. Tat acetylation: A regulatory switch between early and late phases in HIV transcription elongation. Novartis Found Symp 259: 182-193 discussion 193-196, 223-225.

Ottinger M, Christalla T, Nathan K, Brinkmann M, Viejo-Borbolla A, Schulz T. 2006. Kaposi's sarcoma-associated herpesvirus LANA-1 interacts with the short variant of BRD4 and releases cells from a BRD4and BRD2/RING3-induced $\mathrm{G}_{1}$ cell cycle arrest. J Virol 80: 1077210786.

Ottinger M, Pliquet D, Christalla T, Frank R, Stewart JP, Schulz TF. 2009. The interaction of the gammaherpesvirus 68 orf73 protein with cellular BET proteins affects the activation of cell cycle promoters. J Virol 83: $4423-4434$

Pal DK, Evgrafov OV, Tabares P, Zhang F, Durner M, Greenberg DA. 2003. BRD2 (RING3) is a probable major susceptibility gene for common juvenile myoclonic epilepsy. Am J Hum Genet 73: 261-270. 
Pan C, Mezei M, Mujtaba S, Muller M, Zeng L, Li J, Wang Z, Zhou M. 2007. Structure-guided optimization of small molecules inhibiting human immunodeficiency virus 1 Tat association with the human coactivator p300/CREB binding protein-associated factor. $J$ Med Chem 50: 2285-2288.

Parthun MR, Widom J, Gottschling DE. 1996. The major cytoplasmic histone acetyltransferase in yeast: Links to chromatin replication and histone metabolism. Cell 87: 85-94.

Philipps DL, Wigglesworth K, Hartford SA, Sun F, Pattabiraman S, Schimenti K, Handel M, Eppig JJ, Schimenti JC. 2008. The dual bromodomain and WD repeat-containing mouse protein BRWD1 is required for normal spermiogenesis and the oocyte-embryo transition. Dev Biol 317: 72-82.

Poux AN, Marmorstein R. 2003. Molecular basis for Gcn5/PCAF histone acetyltransferase selectivity for histone and nonhistone substrates. Biochemistry 42: 14366-14374.

Poux AN, Cebrat M, Kim CM, Cole PA, Marmorstein R. 2002. Structure of the GCN5 histone acetyltransferase bound to a bisubstrate inhibitor. Proc Natl Acad Sci 99: 14065-14070.

Poveda A, Pamblanco M, Tafrov S, Tordera V, Sternglanz R, Sendra R. 2004. Hif1 is a component of yeast histone acetyltransferase B, a complex mainly localized in the nucleus. J Biol Chem 279: 16033-16043.

Prinjha RK, Witherington J, Lee K. 2012. Place your BETs: The therapeutic potential of bromodomains. Trends Pharmacol Sci 33: 146-153.

* Qi J. 2014. Bromodomain and extraterminal domain inhibitors (BETi) for cancer therapy: Chemical modulation of chromatin structure. Cold Spring Harb Perspect Biol doi: 10.1101/cshperspect.a018663.

Ravindra KC, Selvi BR, Arif M, Reddy BA, Thanuja GR, Agrawal S, Pradhan SK, Nagashayana N, Dasgupta D, Kundu TK. 2009. Inhibition of lysine acetyltransferase KAT3B/p300 activity by a naturally occurring hydroxynaphthoquinone, plumbagin. J Biol Chem 284: 24453-24464.

Renthal W, Nestler EJ. 2009. Histone acetylation in drug addiction. Semin Cell Dev Biol 20: 387-394.

Rojas JR, Trievel RC, Zhou J, Mo Y, Li X, Berger SL, Allis CD, Marmorstein R. 1999. Structure of Tetrahymena GCN5 bound to coenzyme A and a histone H3 peptide. Nature 401: 93-98.

Ruiz-Garcia AB, Sendra R, Galiana M, Pamblanco M, Perez-Ortin JE, Tordera V. 1998. HAT1 and HAT2 proteins are components of a yeast nuclear histone acetyltransferase enzyme specific for free histone $\mathrm{H} 4$. J Biol Chem 273: 12599-12605.

Sachchidanand, Resnick-Silverman L, Yan S, Mutjaba S, Liu WJ, Zeng L, Manfredi JJ, Zhou MM. 2006. Target structure-based discovery of small molecules that block human p53 and CREB binding protein association. Chem Biol 13: 81-90.

Sanchez R, Zhou MM. 2009. The role of human bromodomains in chromatin biology and gene transcription. Curr Opin Drug Discov Devel 12: 659-665.

Sanchez R, Zhou MM. 2011. The PHD finger: A versatile epigenome reader. Trends Biochem Sci 36: 364-372.

Sanchez R, Pieper U, Melo F, Eswar N, Marti-Renom M, Madhusudhan M, Mirkovic N, Sali A. 2000. Protein structure modeling for structural genomics. Nat Struct Biol (suppl.) 7: 986-990.

Sanders BD, Jackson B, Brent M, Taylor AM, Dang W, Berger SL, Schreiber SL, Howitz K, Marmorstein R. 2009. Identification and characterization of novel sirtuin inhibitor scaffolds. Bioorg Med Chem 17: 7031-7041.

Sapountzi V, Cote J. 2011. MYST-family histone acetyltransferases: Beyond chromatin. Cell Mol Life Sci 68: 1147-1156.

* Schaefer U. 2014. Pharmacological inhibition of bromodomain-containing proteins in inflammation. Cold Spring Harb Perspect Biol doi: $10.1101 /$ cshperspect.a018671.

Schultz J, Copley R, Doerks T, Ponting C, Bork P. 2000. SMART: A webbased tool for the study of genetically mobile domains. Nucleic Acids Res 28: 231-234.
* Seto E, Yoshida M. 2014. Erasers of histone acetylation: The histone deacetylase enzymes. Cold Spring Harb Perspect Biol doi: 10.1101/ cshperspect.a018713.

Shang E, Wang X, Wen D, Greenberg DA, Wolgemuth DJ. 2009. Double bromodomain-containing gene $\mathrm{Brd} 2$ is essential for embryonic development in mouse. Dev Dyn 238: 908-917.

Shia WJ, Osada S, Florens L, Swanson SK, Washburn MP, Workman JL. 2005. Characterization of the yeast trimeric-SAS acetyltransferase complex. J Biol Chem 280: 11987-11994.

Smith KT, Workman JL. 2009. Introducing the acetylome. Nat Biotechnol 27: 917-919.

Sonnhammer EL, Eddy SR, Durbin R. 1997. Pfam: A comprehensive database of protein domain families based on seed alignments. Proteins 28: $405-420$.

Spange S, Wagner T, Heinzel T, Kramer OH. 2009. Acetylation of nonhistone proteins modulates cellular signalling at multiple levels. Int $J$ Biochem Cell Biol 41: 185-198.

Spencer TE, Jenster G, Burcin MM, Allis CD, Zhou JX, Mizzen CA, McKenna NJ, Onate SA, Tsai SY, Tsai MJ, et al. 1997. Steroid receptor coactivator-1 is a histone acetyltransferase. Nature 389: 194-198.

Stavropoulos P, Nagy V, Blobel G, Hoelz A. 2008. Molecular basis for the autoregulation of the protein acetyl transferase Rtt109. Proc Natl Acad Sci 105: 12236-12241.

Su D, Hu Q, Zhou H, Thompson JR, Xu RM, Zhang Z, Mer G. 2011. Structure and histone binding properties of the Vps75-Rtt109 chaperone-lysine acetyltransferase complex. J Biol Chem 286: 1562515629.

Sun B, Guo S, Tang Q, Li C, Zeng R, Xiong Z, Zhong C, Ding J. 2011. Regulation of the histone acetyltransferase activity of hMOF via autoacetylation of Lys274. Cell Res 21: 1262-1266.

Sung B, Pandey MK, Ahn KS, Yi T, Chaturvedi MM, Liu M, Aggarwal BB. 2008. Anacardic acid (6-nonadecyl salicylic acid), an inhibitor of histone acetyltransferase, suppresses expression of nuclear factor- $\mathrm{\kappa} B$-regulated gene products involved in cell survival, proliferation, invasion, and inflammation through inhibition of the inhibitory subunit of nuclear factor- $\mathrm{\kappa} \mathrm{B} \alpha$ kinase, leading to potentiation of apoptosis. Blood 111: 4880-4891.

Sutton A, Shia WJ, Band D, Kaufman PD, Osada S, Workman JL, Sternglanz R. 2003. Sas4 and Sas5 are required for the histone acetyltransferase activity of Sas2 in the SAS complex. J Biol Chem 278: 1688716892.

Tang Y, Holbert MA, Wurtele H, Meeth K, Rocha W, Gharib M, Jiang E, Thibault P, Verreault A, Cole PA, et al. 2008. Fungal Rtt109 histone acetyltransferase is an unexpected structural homolog of metazoan p300/CBP. Nat Struct Mol Biol 15: 738-745.

Tang Y, Holbert MA, Delgoshaie N, Wurtele H, Guillemette B, Meeth K, Yuan H, Drogaris P, Lee EH, Durette C, et al. 2011. Structure of the Rtt109-AcCoA/Vps75 complex and implications for chaperonemediated histone acetylation. Structure 19: 221-231.

Tanner KG, Trievel RC, Kuo MH, Howard RM, Berger SL, Allis CD, Marmorstein R, Denu JM. 1999. Catalytic mechanism and function of invariant glutamic acid 173 from the histone acetyltransferase GCN5 transcriptional coactivator. J Biol Chem 274: 18157-18160.

Tanner KG, Langer MR, Denu JM. 2000. Kinetic mechanism of human histone acetyltransferase P/CAF. Biochemistry 39: 15652.

Taunton J, Hassig CA, Schreiber SL. 1996. A mammalian histone deacetylase related to the yeast transcription regulator Rpd3p. Science 272: 408-411.

Taylor SS, Kornev AP. 2011. Protein kinases: Evolution of dynamic regulatory proteins. Trends Biochem Sci 36: 65-77.

Thompson M. 2009. Polybromo-1: The chromatin targeting subunit of the PBAF complex. Biochimie 91: 309-319.

Thompson PR, Wang D, Wang L, Fulco M, Pediconi N, Zhang D, An W, Ge Q, Roeder RG, Wong J, et al. 2004. Regulation of the p300 HAT domain via a novel activation loop. Nat Struct Mol Biol 11: 308-315.

Trievel RC, Rojas JR, Sterner DE, Venkataramani RN, Wang L, Zhou J, Allis CD, Berger SL, Marmorstein R. 1999. Crystal structure and 
mechanism of histone acetylation of the yeast GCN5 transcriptional coactivator. Proc Natl Acad Sci 96: 8931-8936.

Trievel RC, Li FY, Marmorstein R. 2000. Application of a fluorescent histone acetyltransferase assay to probe the substrate specificity of the human p300/CBP-associated factor. Anal Biochem 287: 319-328.

Tsai WW, Wang Z, Yiu TT, Akdemir KC, Xia W, Winter S, Tsai CY, Shi X, Schwarzer D, Plunkett W, et al. 2010. TRIM24 links a non-canonical histone signature to breast cancer. Nature 468: 927-932.

Tsubota T, Berndsen CE, Erkmann JA, Smith CL, Yang L, Freitas MA, Denu JM, Kaufman PD. 2007. Histone H3-K56 acetylation is catalyzed by histone chaperone-dependent complexes. Mol Cell 25: 703-712.

Vandemark AP, Kasten MM, Ferris E, Heroux A, Hill CP, Cairns BR. 2007. Autoregulation of the rsc 4 tandem bromodomain by gen 5 acetylation. Mol Cell 27: 817-828.

Wang L, Tang Y, Cole PA, Marmorstein R. 2008. Structure and chemistry of the p300/CBP and Rtt109 histone acetyltransferases: Implications for histone acetyltransferase evolution and function. Curr Opin Struct Biol 18: 741-747.

Wang Z, Song J, Milne TA, Wang GG, Li H, Allis CD, Patel DJ. 2010. Pro isomerization in MLL1 PHD3-bromo cassette connects H3K4me readout to CyP33 and HDAC-mediated repression. Cell 141: 1183-1194.

Williamson WD, Pinto I. 2012. Histones and genome integrity. Front Biosci 17: 984-995.

Wu J, Wang J, Li M, Yang Y, Wang B, Zheng YG. 2011. Small molecule inhibitors of histone acetyltransferase Tip60. Bioorg Chem 39: 53-58.

Wu H, Moshkina N, Min J, Zeng H, Joshua J, Zhou MM, Plotnikov AN. 2012. Structural basis for substrate specificity and catalysis of human histone acetyltransferase 1. Proc Natl Acad Sci 109: 8925-8930.

Xi Q, Wang Z, Zaromytidou AI, Zhang XH, Chow-Tsang LF, Liu JX, Kim H, Barlas A, Manova-Todorova K, Kaartinen V, et al. 2011. A poised chromatin platform for TGF- $\beta$ access to master regulators. Cell 147: $1511-1524$.

Yan Y, Barlev NA, Haley RH, Berger SL, Marmorstein R. 2000. Crystal structure of yeast Esal suggests a unified mechanism of catalysis and substrate binding by histone acetyltransferases. Mol Cell 6: 1195-1205.

Yan Y, Harper S, Speicher DW, Marmorstein R. 2002. The catalytic mechanism of the ESA1 histone acetyltransferase involves a self-acetylated intermediate. Nature Struct Biol 9: 862-869.

Yap KL, Zhou MM. 2010. Keeping it in the family: Diverse histone recognition by conserved structural folds. Crit Rev Biochem Mol Biol 45: 488-505.

Yuan H, Rossetto D, Mellert H, Dang W, Srinivasan M, Johnson J, Hodawadekar S, Ding EC, Speicher K, Abshiru N, et al. 2012. MYST protein acetyltransferase activity requires active site lysine autoacetylation. $E M B O J$ 31: 58-70.
Zeng L, Li J, Muller M, Yan S, Mujtaba S, Pan C, Wang Z, Zhou M. 2005. Selective small molecules blocking HIV-1 Tat and coactivator PCAF association. J Am Chem Soc 127: 2376-2377.

Zeng L, Yap KL, Ivanov AV, Wang X, Mujtaba S, Plotnikova O, Rauscher FJ 3rd, Zhou MM. 2008a. Structural insights into human KAP1 PHD finger-bromodomain and its role in gene silencing. Nat Struct Mol Biol 15: $626-633$.

Zeng L, Zhang Q, Gerona-Navarro G, Moshkina N, Zhou MM. 2008b. Structural basis of site-specific histone recognition by the bromodomains of human coactivators $\mathrm{PCAF}$ and $\mathrm{CBP} / \mathrm{p} 300$. Structure 16: $643-652$.

Zeng L, Zhang Q, Li S, Plotnikov AN, Walsh MJ, Zhou MM. 2010. Mechanism and regulation of acetylated histone binding by the tandem PHD finger of DPF3b. Nature 466: 258-262.

Zhang J, Sprung R, Pei J, Tan X, Kim S, Zhu H, Liu CF, Grishin NV, Zhao Y. 2009. Lysine acetylation is a highly abundant and evolutionarily conserved modification in Escherichia coli. Mol Cell Proteomics 8: 215-225.

Zhang Q, Chakravarty S, Ghersi D, Zeng L, Plotnikov AN, Sanchez R, Zhou MM. 2010. Biochemical profiling of histone binding selectivity of the yeast bromodomain family. PLoS One 5: e8903.

Zhang G, Liu R, Zhong Y, Plotnikov AN, Zhang W, Zeng L, Rusinova E, Gerona-Nevarro G, Moshkina N, Joshua J, et al. 2012. Down-regulation of NF- $\mathrm{BB}$ transcriptional activity in HIV-associated kidney disease by BRD4 inhibition. J Biol Chem 287: 28840-28851.

Zhao R, Nakamura T, Fu Y, Lazar Z, Spector DL. 2011. Gene bookmarking accelerates the kinetics of post-mitotic transcriptional re-activation. Nat Cell Biol 13: 1295-1304.

Zhou M, Huang K, Jung KJ, Cho WK, Klase Z, Kashanchi F, Pise-Masison CA, Brady JN. 2009. Bromodomain protein Brd4 regulates human immunodeficiency virus transcription through phosphorylation of CDK9 at threonine 29. J Virol 83: 1036-1044.

Zou JX, Revenko AS, Li LB, Gemo AT, Chen HW. 2007. ANCCA, an estrogen-regulated AAA + ATPase coactivator for $\mathrm{ER} \alpha$, is required for coregulator occupancy and chromatin modification. Proc Natl Acad Sci 104: 18067-18072.

Zou JX, Guo L, Revenko AS, Tepper CG, Gemo AT, Kung HJ, Chen HW. 2009. Androgen-induced coactivator ANCCA mediates specific androgen receptor signaling in prostate cancer. Cancer Res 69: 33393346.

Zuber J, Shi J, Wang E, Rappaport AR, Herrmann H, Sison EA, Magoon D, Qi J, Blatt K, Wunderlich M, et al. 2011. RNAi screen identifies Brd4 as a therapeutic target in acute myeloid leukaemia. Nature 478: 524528 


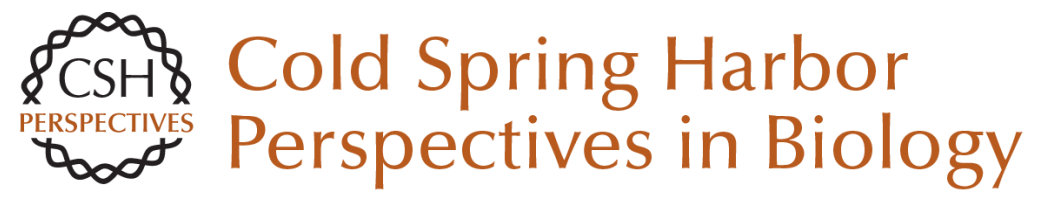

\section{Writers and Readers of Histone Acetylation: Structure, Mechanism, and Inhibition}

Ronen Marmorstein and Ming-Ming Zhou

Cold Spring Harb Perspect Biol 2014; doi: 10.1101/cshperspect.a018762

\section{Subject Collection Epigenetics}

Metabolic Signaling to Chromatin

Shelley L. Berger and Paolo Sassone-Corsi

Histone and DNA Modifications as Regulators of

Neuronal Development and Function Stavros Lomvardas and Tom Maniatis

Histone Modifications and Cancer James E. Audia and Robert M. Campbell

Epigenetics and Human Disease Huda Y. Zoghbi and Arthur L. Beaudet

Induced Pluripotency and Epigenetic Reprogramming

Konrad Hochedlinger and Rudolf Jaenisch

\section{Long-Range Chromatin Interactions} Job Dekker and Tom Misteli

RNAi and Heterochromatin Assembly Robert Martienssen and Danesh Moazed

Dosage Compensation in Drosophila John C. Lucchesi and Mitzi I. Kuroda
Epigenetic Determinants of Cancer Stephen B. Baylin and Peter A. Jones

Maintenance of Epigenetic Information Geneviève Almouzni and Howard Cedar

A Structural Perspective on Readout of Epigenetic Histone and DNA Methylation Marks Dinshaw J. Patel

The Necessity of Chromatin: A View in

Perspective Vincenzo Pirrotta

Germline and Pluripotent Stem Cells Wolf Reik and M. Azim Surani

Comprehensive Catalog of Currently Documented Histone Modifications Yingming Zhao and Benjamin A. Garcia

Epigenetic Regulation of Chromatin States in Schizosaccharomyces pombe Robin C. Allshire and Karl Ekwall

Histone Variants and Epigenetics Steven Henikoff and M. Mitchell Smith

For additional articles in this collection, see http://cshperspectives.cshlp.org/cgi/collection/

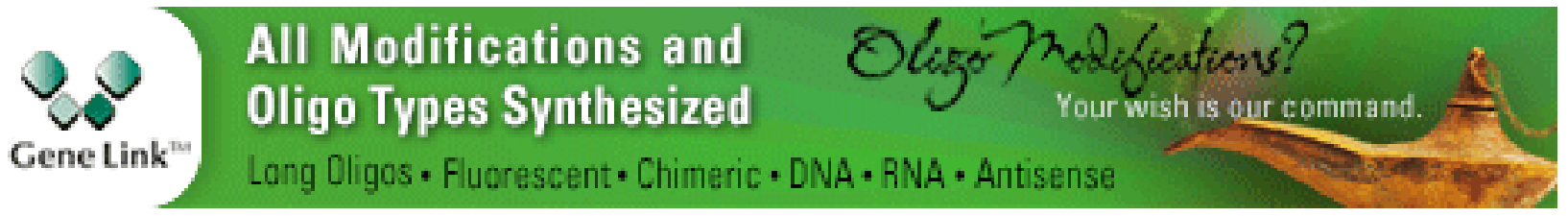


For additional articles in this collection, see http://cshperspectives.cshlp.org/cgi/collection/

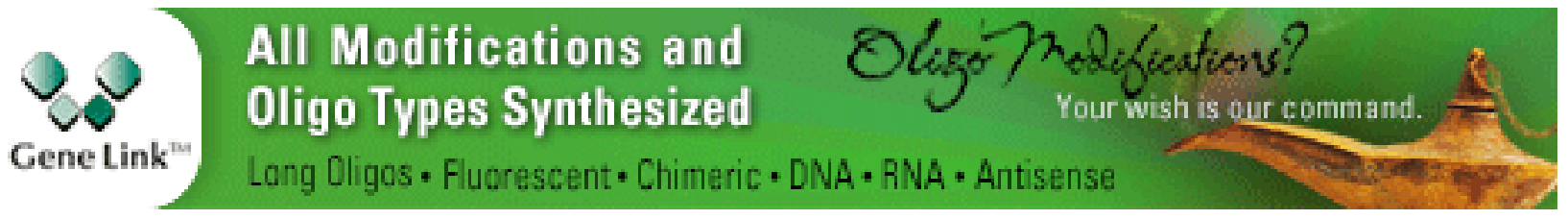

Copyright @ 2014 Cold Spring Harbor Laboratory Press; all rights reserved 